\title{
Docetaxel Reverses Pulmonary Vascular Remodeling by Decreasing Autophagy and Resolves Right Ventricular Fibrosis
}

\author{
Yasmine F. Ibrahim, ${ }^{1}$ Nataliia V. Shults, ${ }^{1}$ Vladyslava Rybka, and Yuichiro J. Suzuki \\ Department of Pharmacology and Physiology, Georgetown University Medical Center, Washington, DC (Y.F.I., N.V.S., V.R., \\ Y.J.S.); and Department of Pharmacology, Minia University School of Medicine, Minia, Egypt (Y.F.I.)
}

Received January 8, 2017; accepted July 19, 2017

\begin{abstract}
Pulmonary arterial hypertension remains a fatal disease despite the availability of approved vasodilators. Since vascular remodeling contributes to increased pulmonary arterial pressure, new agents that reduce the thickness of pulmonary vascular walls have therapeutic potential. Thus, antitumor agents that are capable of killing cells were investigated. Testing of various antitumor drugs identified that docetaxel is a superior drug for killing proliferating pulmonary artery smooth muscle cells compared with other drugs, including gemcitabine, methotrexate, and ifosfamide. The administration of docetaxel to rats with severe pulmonary arterial hypertension reversed pulmonary vascular remodeling and reduced right ventricular pressure. Docetaxel was found to decrease autophagy as monitored by LC3B-II and p62 expression. The small interfering RNA knockdown
\end{abstract}

of Beclin-1 or LC3B potentiated docetaxel-induced cell death, and knocking down p62 inhibited the docetaxel effects. The suppressed autophagic process is due to the ability of docetaxel to decrease Beclin-1 protein expression in a proteasomedependent manner. Mass spectrometry identified a novel docetaxel-inducible Beclin-1 binding protein, namely, myosin-9. Knocking down myosin-9 inhibited docetaxel-induced cell death. In damaged right ventricles of pulmonary arterial hypertension rats, docetaxel remarkably promoted the resolution of fibrosis and the regeneration of myocardium. Thus, docetaxel is capable of reversing pulmonary vascular remodeling and resolving right ventricle fibrosis and is a promising therapeutic agent for the treatment of pulmonary arterial hypertension and right heart failure.

\section{Introduction}

In pulmonary arterial hypertension (PAH), pulmonary artery (PA) resistance is increased due to vasoconstriction and vascular remodeling (Thompson and Lawrie, 2017). PAH remains a fatal disease without a cure (Peacock et al., 2007; Galiè et al., 2009). Increased resistance in the pulmonary circulation strains the right ventricle (RV), leading to rightsided heart failure and death. The National Institutes of Health registry determined that, if patients are not treated, the median survival duration of $\mathrm{PAH}$ patients after diagnosis is 2.8 years, with the 3 -year survival being $48 \%$ (D'Alonzo et al., 1991). Since then, vasodilatory drugs affecting three pathways (prostacyclin, endothelin, and nitric oxide) have become available to treat PAH. These drugs improve the quality of lives of patients; however, their influence on survival is minimal. Even

This work was supported by the National Institutes of Health National Heart, Lung, and Blood Institute and National Institute of Aging [Grants R01 HL72844 and R03 AG047824 to Y.J.S.]. The content is solely the responsibility of the authors and does not necessarily represent the official views of the National Institutes of Health.

${ }^{1}$ Y.F.I. and N.V.S. contributed equally to this work.

https://doi.org/10.1124/jpet.117.239921. with currently available therapies, the prognosis is poor, with 3 -year survival being reported to be $58 \%-75 \%$ (Benza et al., 2010; Humbert et al., 2010; Thenappan et al., 2010; Chung et al., 2014; Jansa et al., 2014; Olsson et al., 2014). Thus, the development of improved therapeutic strategies is warranted for the treatment of this disease.

The major function of these approved drugs is to promote vasodilation. However, since the growth of vascular cells is also critical to the elevation of vascular resistance, agents that eliminate excess vascular cells should have therapeutic potential by reducing the thickness of the pulmonary vascular walls, which has often already increased by the time patients are diagnosed (Archer and Michelakis, 2006). In this regard, cancer chemotherapeutic drugs with abilities to kill cells may be useful in the treatment of PAH (Suzuki et al., 2007).

We have previously shown that antitumor drugs, including anthracyclines and proteasome inhibitors, are effective at reversing PAH by reducing PA wall thickening (Ibrahim et al., 2014; Wang et al., 2016). These agents were found to selectively cause apoptotic and autophagic death of cells in the remodeled pulmonary vasculature of animals with PAH, but not in normal vessels of animals without the disease.

ABBREVIATIONS: DTX, docetaxel; MG132, (Benzyl N-[(2S)-4-methyl-1-[(2S)-4-methyl-1-[[(2S)-4-methyl-1-oxopentan-2-yl]amino]-1-oxopentan2-yl]amino]-1-oxopentan-2-yl]carbamate); MYH9, myosin-9; PA, pulmonary artery; PAEC, pulmonary artery endothelial cell; PAH, pulmonary arterial hypertension; PASMC, pulmonary artery smooth muscle cell; RV, right ventricle; SBI-0206965, (2-((5-Bromo-2-((3,4,5-trimethoxyphenyl)amino)pyrimidin-4yl)oxy)-N-methylbenzamide); siRNA, small interfering RNA; SU5416, (1,3-Dihydro-3-[(3,5-dimethyl-1H-pyrrol-2-yl)methylene]-2H-indol-2-one); TUNEL, terminal deoxynucleotidyl transferase-mediated digoxigenin-deoxyuridine nick-end labeling; Z-VAD-FMK, (N-Benzyloxycarbonyl-Val-AlaAsp(O-Me) fluoromethyl ketone). 
These drugs, however, are known to cause cardiotoxicity (Minotti et al., 2004; Bockorny et al., 2012; Gupta et al., 2012; Menna et al., 2012), which may limit use in PAH patients with a weakened heart.

To find better drugs, the present study first examined if other antitumor drugs are also effective at killing pulmonary vascular cells. We identified that docetaxel (DTX) is a potent drug that can kill cultured proliferating human PA smooth muscle cells (PASMCs) and PA endothelial cells (PAECs). DTX is a drug that is a member of the taxane drug class, which disrupts microtubule functions, thereby inhibiting cell division (Fojo and Menefee, 2007). DTX is clinically used for treating locally advanced or metastatic breast cancer, head and neck cancer, gastric cancer, hormone-refractory prostate cancer, and non-small-cell lung cancer (Gligorov and Lotz, 2004). The present study tested the effects of DTX on pulmonary vascular remodeling in rats with PAH. We found that DTX not only reverses pulmonary vascular remodeling, but also remarkably repairs the failing $\mathrm{RV}$.

\section{Materials and Methods}

Cell Culture Experiments. Human PASMCs and PAECs were purchased from ScienCell Research Laboratories (Carlsbad, CA) and Cell Applications, Inc. (San Diego, CA) and were cultured in accordance with the manufacturers' instructions in $5 \% \quad \mathrm{CO}_{2}$ at $37^{\circ} \mathrm{C}$. Experimental results were confirmed in cells from multiple donors, and by the time this study was completed, cells from eight different individuals were purchased. Cells in passages 3-7 were used. Differentiated PASMCs were generated by using the Differentiation Medium from Cell Applications in accordance with the manufacturer's instructions. HeLa human cervical cancer cells were obtained from the Lombardi Comprehensive Cancer Center Tissue Culture Shared Resources at Georgetown University (Washington, DC). For small interfering RNA (siRNA) knockdown, cells were transfected with an siRNA Transfection Reagent and gene silencing siRNA or control siRNA with a scrambled sequence (Santa Cruz Biotechnology, Dallas, TX). Cells were used for experiments 2 days after the transfection. In some experiments, Beclin-1 was overexpressed by treating cells with adenovirus expressing human Beclin-1 (Vector Biosystems Inc., Eagleville, PA). Cells were treated with gemcitabine hydrochloride, methotrexate, ifosfamide, DTX, bortezomib, MG132 (Benzyl N-[(2S)-4-methyl-1-[[(2S)4-methyl-1-[[(2S)-4-methyl-1-oxopentan-2-yl]amino]-1-oxopentan2-yl]amino]-1-oxopentan-2-yl]carbamate), paclitaxel, vincristine, carfilzomib, SBI-0206965 (2-((5-Bromo-2-((3,4,5-trimethoxyphenyl)amino)pyrimidin4-yl)oxy)-N-methylbenzamide), or Z-VAD-FMK (N-BenzyloxycarbonylVal-Ala-Asp(O-Me) fluoromethyl ketone) (Selleckchem, Houston, TX) dissolved in dimethylsulfoxide or daunorubicin hydrochloride (SigmaAldrich, St. Louis, MO) dissolved in water. Equal amounts of dimethylsulfoxide were included in controls. The number of viable cells was determined by using Cell Counting Kit-8 (Dojindo Molecular Technologies, Rockville, MD) or by counting on a hemocytometer.

Animal Experiments. The present study used the SU5416 (1,3Dihydro-3-[(3,5-dimethyl-1H-pyrrol-2-yl)methylene]-2H-indol-2-one)/ hypoxia model with pathologic features similar to those in human $\mathrm{PAH}$ (Taraseviciene-Stewart et al., 2001; Oka et al., 2007; Abe et al., 2010; Ibrahim et al., 2014; Wang et al., 2016). Male Sprague-Dawley rats ( $250 \mathrm{~g}$; Charles River Laboratories, Wilmington, MA) were subcutaneously injected with $20 \mathrm{mg} / \mathrm{kg}$ body weight SU5416 (TOCRIS, Minneapolis, MN), maintained in hypoxia for 3 weeks, then in normoxia. Animals were subjected to sustained hypoxia in a chamber regulated by an OxyCycler Oxygen Profile Controller (BioSpherix, Redfield, NY) that maintains $10 \% \mathrm{O}_{2}$ with an influx of $\mathrm{N}_{2}$ gas (Park et al., 2010; Ibrahim et al., 2014; Wang et al., 2016).

After pulmonary hypertension and pulmonary vascular remodeling developed, rats were then injected intraperitoneally with pharmaceuticalgrade DTX ( $5 \mathrm{mg} / \mathrm{kg}$ body weight; Sagent Pharmaceuticals, Schaumburg, IL). After 4 days, rats were injected again with DTX (5 mg/kg). After

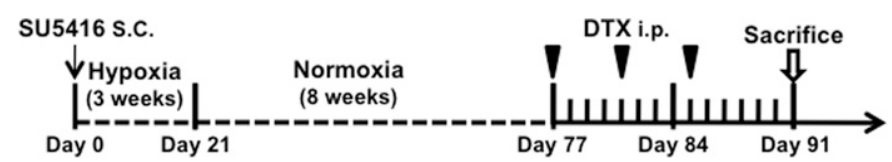

Scheme 1. Schematics of animal experiments. On day 0, rats were injected s.c. with SU5416 (20 mg/kg body weight) and placed in an OxyCycler chamber to be subjected to chronic hypoxia at $10 \% \mathrm{O}_{2}$ for 3 weeks. On day 21 , rats were then placed in normoxia for 8 weeks to promote the development of pulmonary hypertension and pulmonary vascular remodeling. On day 77, rats were then injected i.p. with DTX ( $5 \mathrm{mg} / \mathrm{kg}$ body weight). After 4 days, on day 81 , rats were again injected with DTX ( $5 \mathrm{mg} / \mathrm{kg}$ body weight). After another 4 days, on day 85, rats were injected with DTX (6 mg/kg body weight). Animals were kept in normoxia for an additional 6 days.

another 4 days, rats were injected with DTX (6 mg/kg). Control animals were injected with equal amounts of saline. Animals were placed back in normoxia for an additional 6 days before hemodynamic measurements and the lung and heart harvest (Scheme 1).

For hemodynamic measurements, rats were anesthetized with intraperitoneal injections of urethane ( $1.6 \mathrm{~g} / \mathrm{kg}$ body weight). Animals were then intubated and mechanically ventilated with a volumecontrolled Inspira Advanced Safety Ventilator (Harvard Apparatus, Holliston, MA). Rats were maintained on a heat pad, and the temperature was kept at $37^{\circ} \mathrm{C}$ using a TR-200 Temperature Controller connected to a rectal probe (Fine Scientific Tools, North Vancouver, Canada). After a thoracotomy, a Millar catheter (1.4 F; Millar, Houston, TX) was inserted into the RV. RV pressure signals were recorded by using PowerLab with Chart 5 software (ADInstruments, Colorado Springs, CO). Extrapulmonary arteries (left and right main branches) and intrapulmonary arteries (first-order branch) were surgically dissected, and connective tissues were gently removed in ice-cold phosphate-buffered saline under a dissecting microscope.

Georgetown University Animal Care and Use Committee approved all animal experiments. The investigation conformed to the National Institutes of Health Guide for the Care and Use of Laboratory Animals.

Histologic Measurements. Tissues were immersed in buffered $10 \%$ formalin at room temperature, and were embedded in paraffin. Paraffin-embedded tissues were cut and mounted on glass slides. Tissue sections were stained with H\&E. Lung slides were analyzed for PA wall thickness and vessel radius. Ten to 15 vessels were analyzed per animal, and six values for thickness and two values for radius were measured for each vessel. The percentage wall thickness values, defined as wall thickness divided by vessel radius, were calculated. Tissue sections were also evaluated for smooth muscle mass by immunohistochemistry by using the $\alpha$-smooth muscle actin antibody (Abcam, Cambridge, UK), fibrosis by Masson's trichrome stain, and apoptosis by terminal deoxynucleotidyl transferase-mediated digoxigenin-deoxyuridine nick-end labeling (TUNEL) assay. TUNEL assay was performed by using the ApopTag kit (EMD Millipore, Billerica, MA) with minor modifications. In brief, heat-induced epitope retrieval was performed by immersing the deparaffinized tissues. Slides were exposed to terminal transferase and digoxigenin-labeled dUTP, blocked, treated with horseradish peroxidase-conjugated antidigoxigenin antibody and DAB (3,3'-Diaminobenzidine) chromagen, counterstained with hematoxylin, and mounted with Acrymount (StatLab, McKinney, TX). Percentage apoptotic cells was calculated by dividing the number of apoptotic cells by the total number of cells.

Western Blotting. Equal protein amounts of samples were electrophoresed through a reducing SDS polyacrylamide gel and electroblotted onto a membrane. The membrane was blocked and incubated with antibodies for LC3B (Cell Signaling Technology, Danvers, MA), myosin-9 (MYH9), Beclin-1 (Santa Cruz Biotechnology), and p62 (Syd Laboratories, Inc., Malden, MA), and levels of proteins were detected by using horseradish peroxidase-linked secondary antibodies and an Enhanced Chemiluminescence System (Amersham Life Science, Arlington Heights, IL). Seventy-microgram tissue homogenate proteins were used for LC3B, and 50- $\mu \mathrm{g}$ tissue homogenate proteins were used for Beclin-1, p62, and 

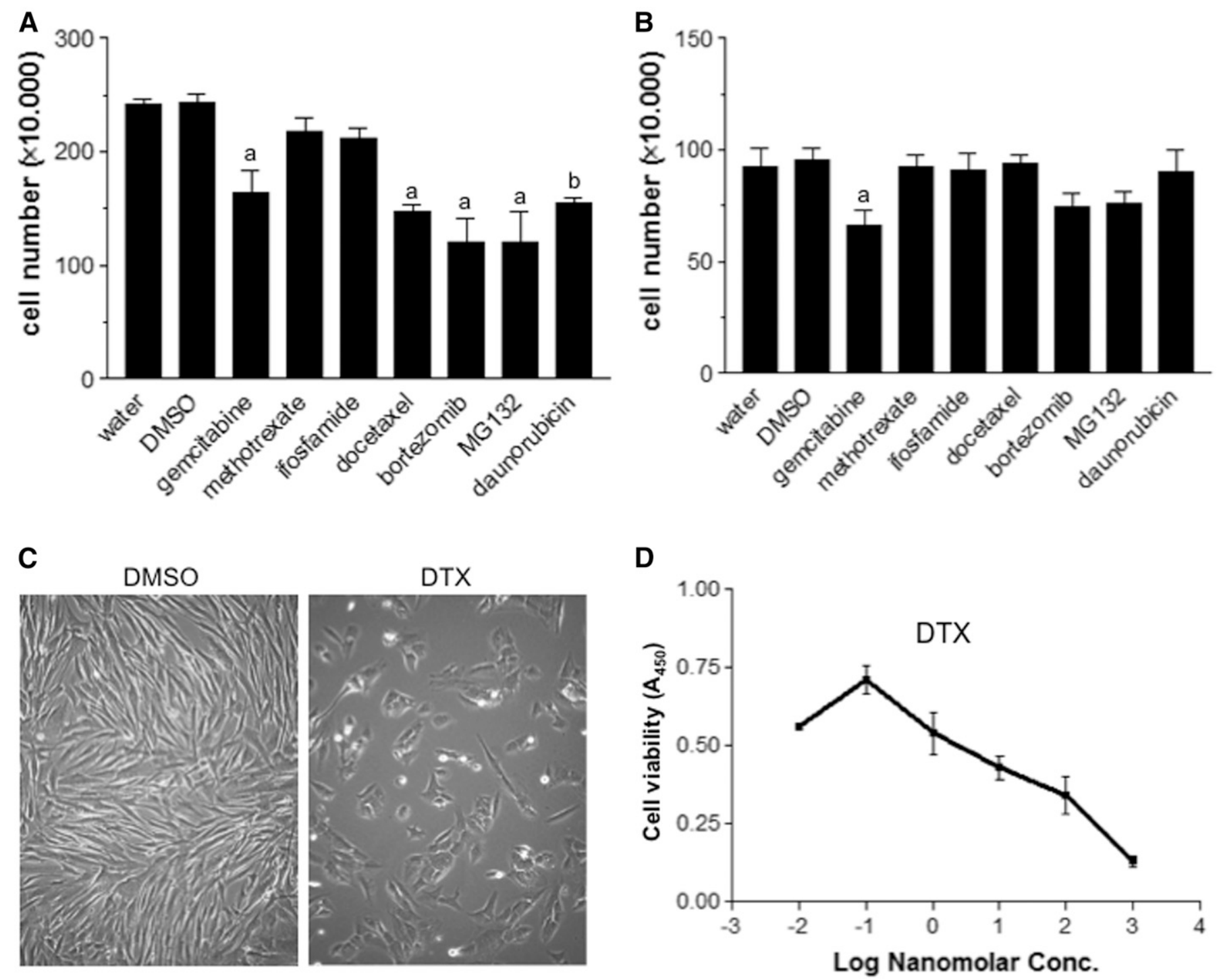

E

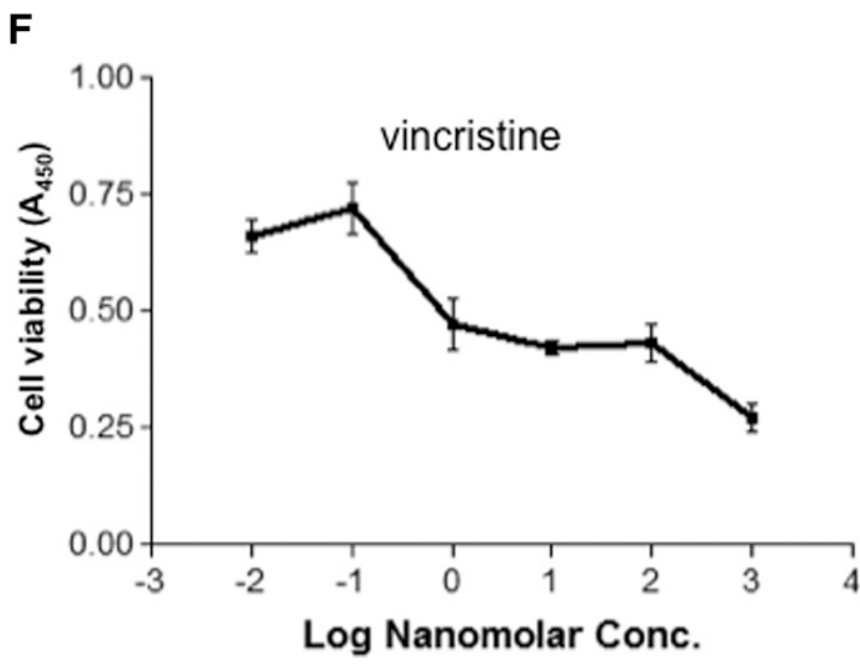

Fig. 1. Microtubule-disturbing drugs, including DTX, are effective in killing proliferating PASMCs. Proliferating/synthetic phenotype (A) and differentiated/contractile phenotype of human PASMCs (B) were treated with various antitumor drugs at $1 \mu \mathrm{M}$ for 24 hours. Cell number was determined by counting on a hemocytometer. Equal amounts of water (for daunorubicin) and $0.1 \%$ dimethylsulfoxide (DMSO; for other drugs) were used as vehicle controls. Symbols a and b denote significantly different from DMSO and water, respectively $(n=6-9)$ at $P<0.05$. (C) Representative photographs of control and DTX-treated PASMCs. (D-F) Proliferating/synthetic human PASMCs were treated with DTX, paclitaxel, or vincristine for 24 hours. The number of viable cells was monitored by using Cell Counting Kit-8. 
A

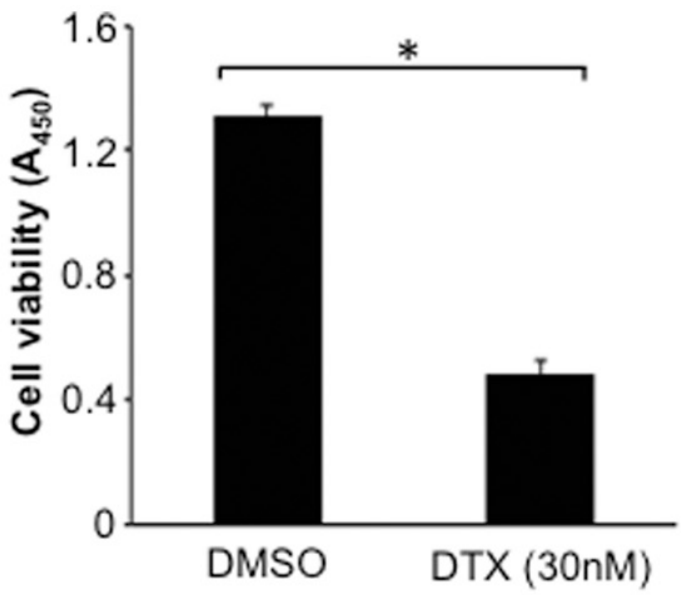

B

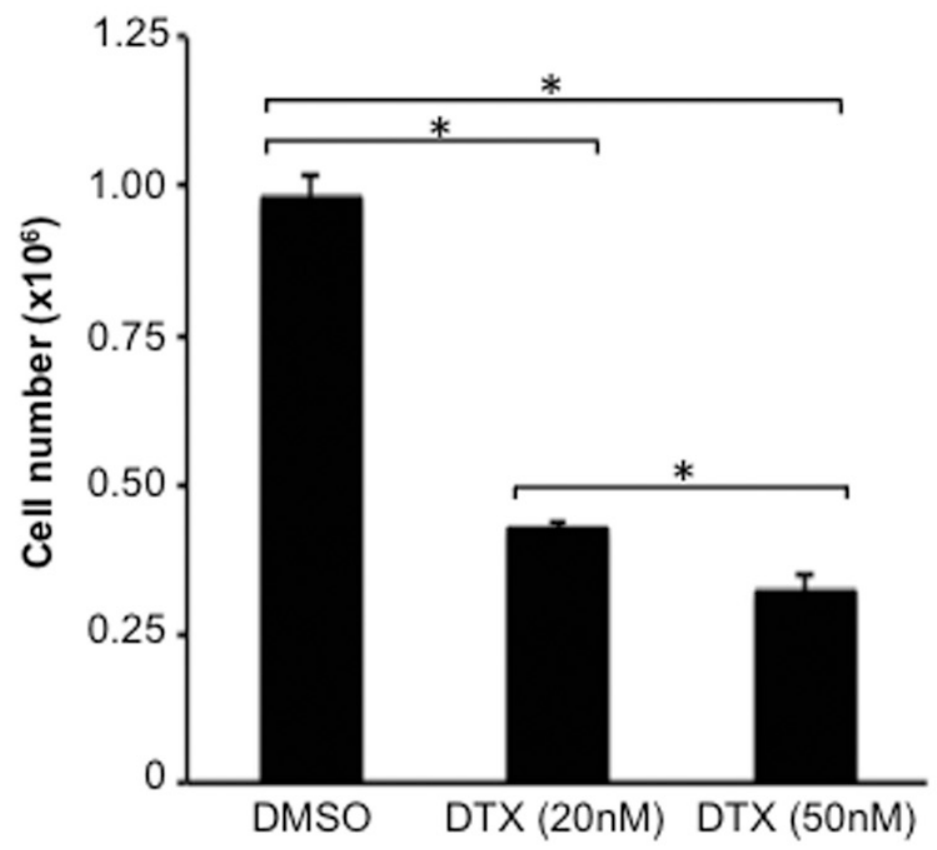

C

DMSO

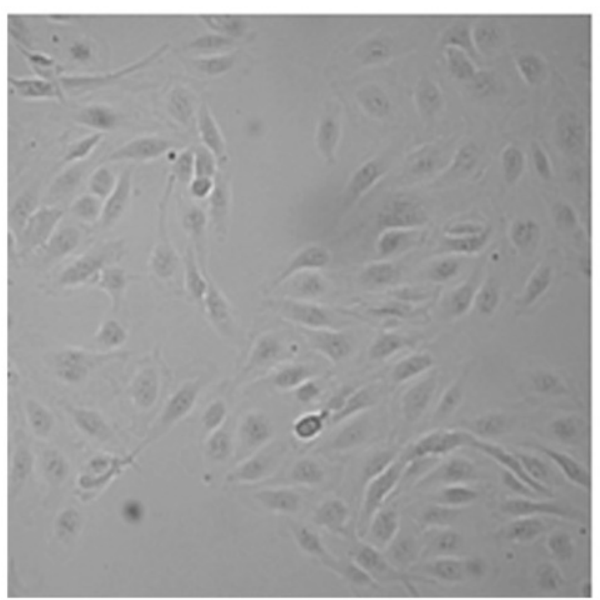

DTX (50nM)

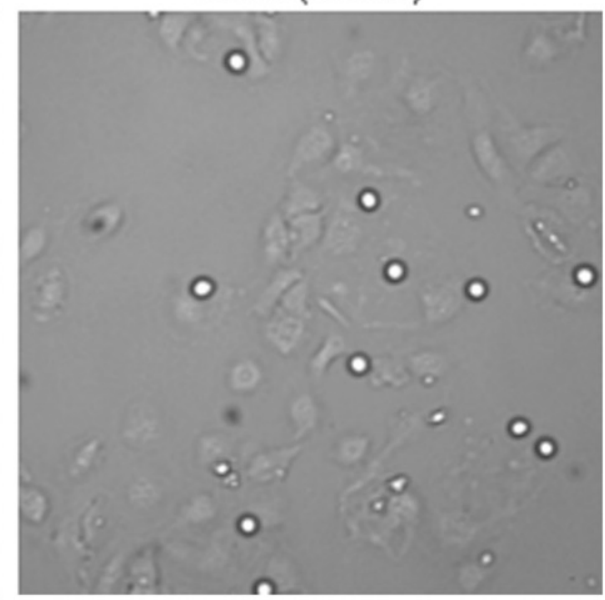

Fig. 2. Effects of DTX on human PAECs. Human PAECs were treated with DTX for 24 hours. (A) The number of viable cells was monitored by using Cell Counting Kit-8 $(n=16)$. (B) The number of cells was counted on a hemocytometer $(n=4)$. ${ }^{*}$ The two values are significantly different from each other at $P<0.05$. (C) Representative photographs of control and DTX-treated PAECs. DMSO, dimethylsulfoxide. 
A

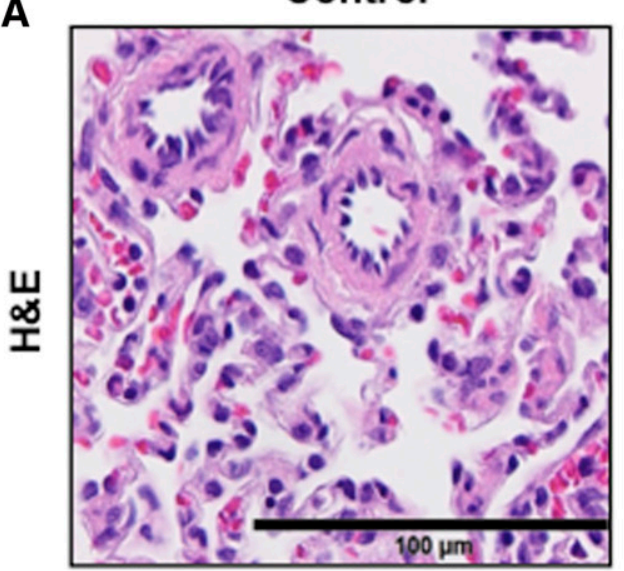

Control

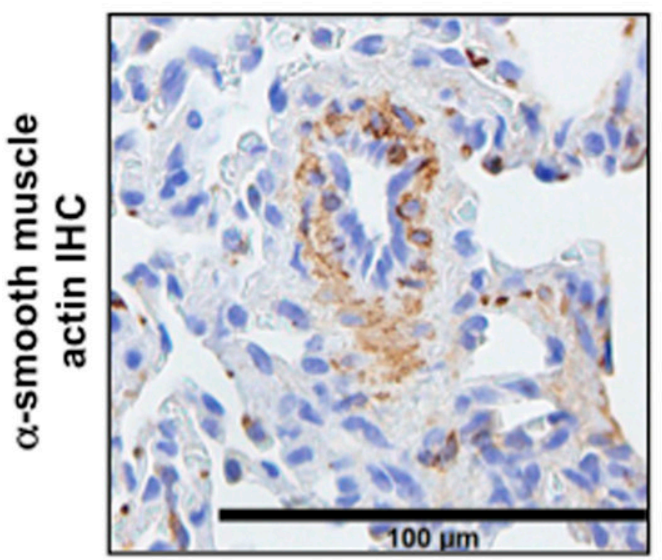

PAH
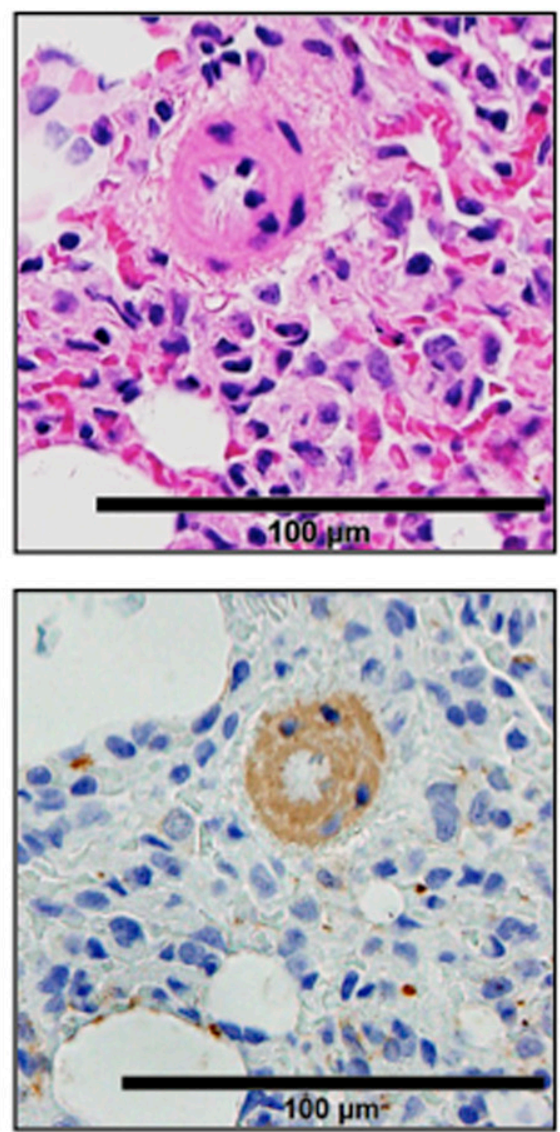

PAH+DTX
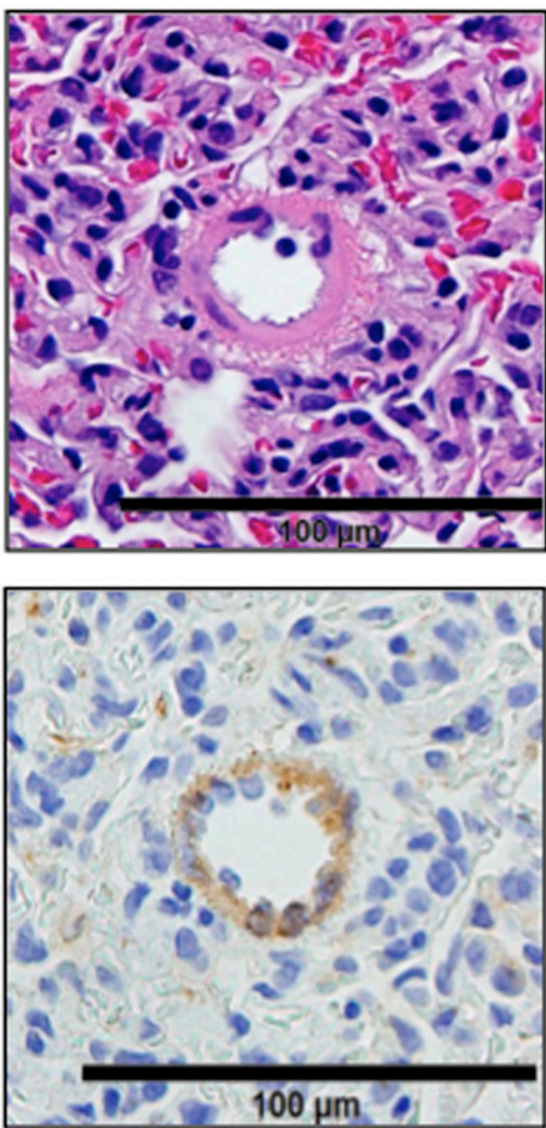

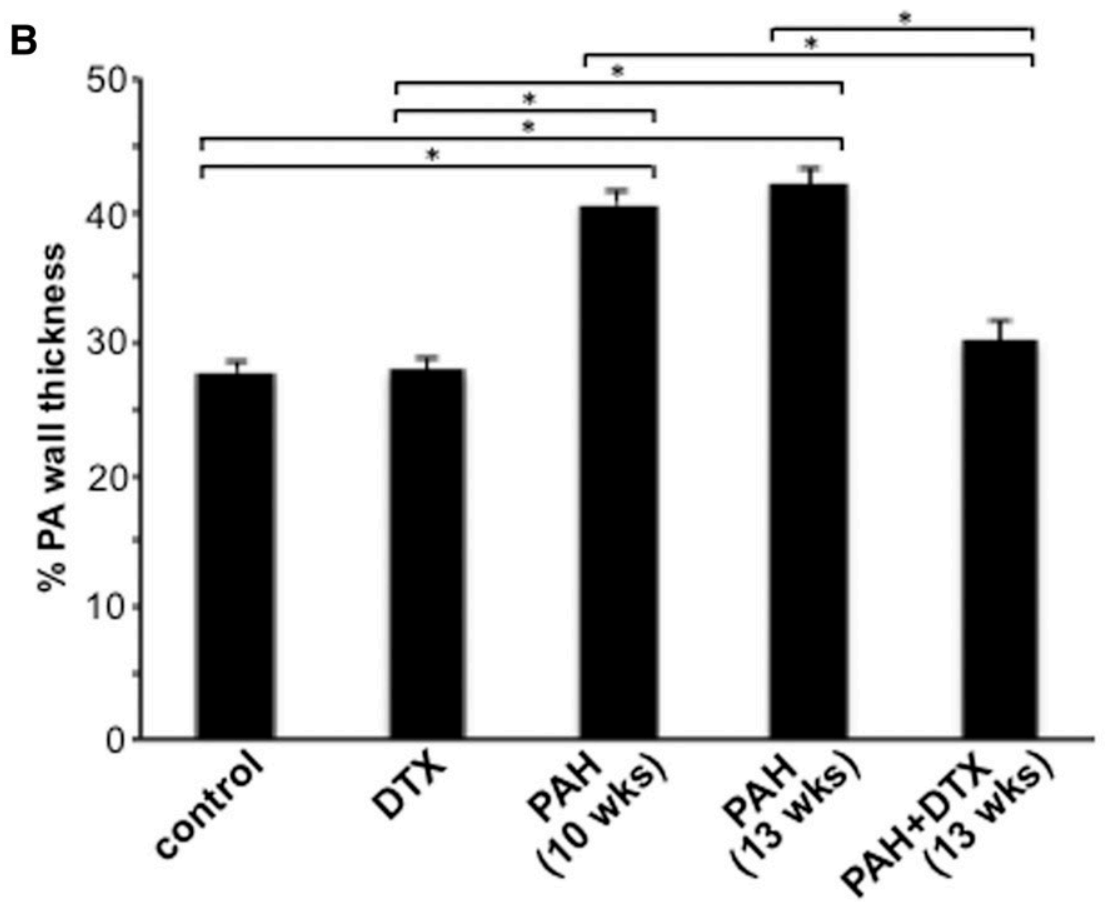

Fig. 3. DTX reverses PA remodeling in experimental animals. On day 70 ( 10 weeks) and day 91 ( 13 weeks), rats were euthanized. Lungs were harvested, immersed in buffered 10\% formalin, and embedded in paraffin for H\&E staining and immunohistochemistry (IHC) using the $\alpha$-smooth muscle actin antibody. (A) Representative images at $\times 400$ magnification are shown. (B) Percentage PA wall thickness was calculated in H\&E-stained sections. The bar graph represents means \pm S.E.M. $(n=6-7)$ of percentage PA wall thickness of pulmonary arterioles/small PA of diameters ranging from 29.5 to $78.5 \mu \mathrm{m}$ (mean diameter $58.1 \mu \mathrm{m}$ ). *The two values are significantly different from each other at $P<0.05$. 


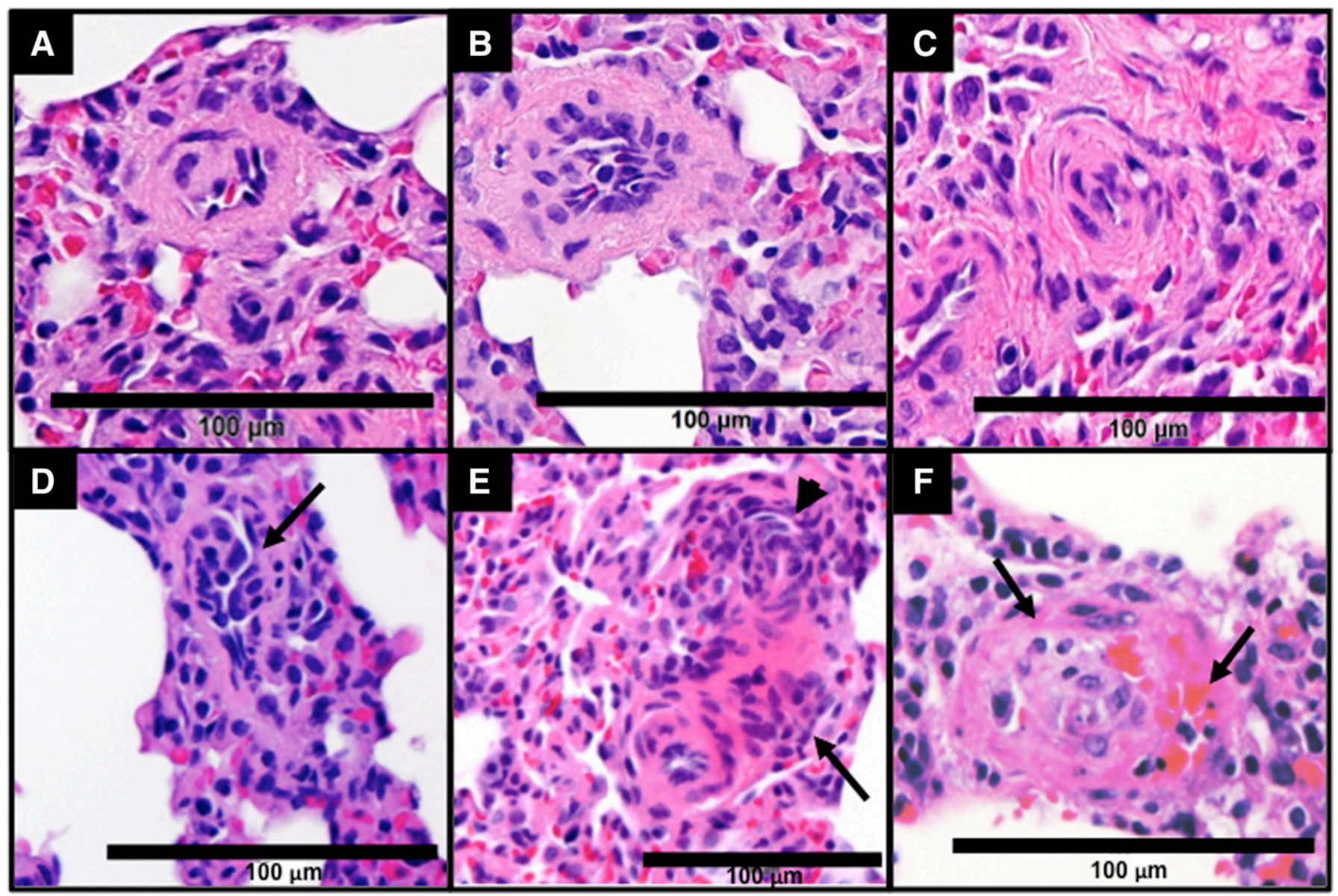

Fig. 4. Different types of pulmonary vascular lesions found in the lungs of PAH rats. Rats were treated with SU5416 and sustained hypoxia (3 weeks) and then maintained in normoxia for 10 weeks. Lungs were harvested, immersed in buffered $10 \%$ formalin, and embedded in paraffin. H\&E staining shows remodeled arterioles with increased wall thickness (A), intimal cell proliferation (B), concentric occlusive lesions (C), and plexiform lesions (D-F). (D) Proliferation of endothelial cells forming slit-like channels (arrow). (E) An illustration of the multiple concentric onion-skin pattern (arrowhead) and conglomerate of endothelial cells, perhaps consistent with a partial slice through a plexiform lesion (arrow). (F) The concentric-obliterative lesion multichanneled cellular lesions (arrows) $(\times 400)$.

MYH9. Twenty-microgram cell lysate proteins were used for LC3B and MYH9, and 15- $\mu$ g cell lysate proteins were used for Beclin-1 and p62.

Immunoprecipitation. Cell lysates were incubated with Beclin-1 antibody, MYH9 antibody, or normal IgG (Santa Cruz) and GammaBind G-Sepharose (Amersham) overnight at $4^{\circ} \mathrm{C}$ with gentle shaking. Samples were centrifuged, and the pellets were washed twice with ice-cold lysis buffer without Triton X-100 and boiled in Laemmli buffer for 5 minutes, followed by centrifugation. The supernatants were subjected to SDS polyacrylamide gel electrophoresis. Gels were either stained with Coomassie Blue or immunoblotted with MYH9 or Beclin-1 antibody.

Statistical Analysis. Means and standard errors were calculated. Comparisons between two groups were analyzed by using a two-tailed Student's $t$ test, and comparisons between more than two groups were analyzed by analysis of variance with a Student-Newman-Keuls posthoc test using GraphPad Prism (GraphPad Software, Inc., La Jolla, $\mathrm{CA})$ in accordance with the Kolmogorov-Smirnov test for normality. $P<0.05$ was considered to be significant.

\section{Results}

Effects of Various Antitumor Drugs on Pulmonary Vascular Cells. We have previously shown that an anthracycline cancer chemotherapeutic agent (daunorubicin) and proteasome inhibitors (MG132, bortezomib, and carfilzomib) can effectively reverse pulmonary vascular remodeling, suggesting that antitumor drugs may be useful for the treatment of PAH (Ibrahim et al., 2014; Wang et al., 2016). To further identify candidate drugs to be used in the PAH treatment, the present study tested various antitumor drugs for killing cultured proliferating/synthetic human PASMCs, the phenotype relevant to cells in the remodeled pulmonary vasculature (Ibrahim et al., 2014). Daunorubicin, bortezomib, MG132, gemcitabine, and DTX were found to effectively kill PASMCs (Fig. 1A). By contrast, methotrexate and ifosfamide were ineffective at killing proliferating PASMCs (Fig. 1A). Gemcitabine, but no other drugs, also caused the significant death of differentiated/contractile PASMCs that may resemble the functional SMCs of the pulmonary vasculature, which should be preserved (Fig. 1B). Thus, DTX was determined to be a promising drug to be further investigated.

Figure 1C shows representative photographs of control proliferating PASMCs and cells treated with DTX. Not only was the DTX-treated cell population less than controls (including before the DTX treatment), DTX-treated PASMCs were found to be round compared with long and thin control cells. The primary action of DTX and the other taxane family of drugs is to inhibit the depolymerization of microtubules by stabilizing microtubules. Dose-response experiments showed that DTX kills proliferating human PASMCs, with an $\mathrm{IC}_{50}$ of $\sim 10 \mathrm{nM}$ (Fig. 1D). The efficacy of DTX to kill human 
A

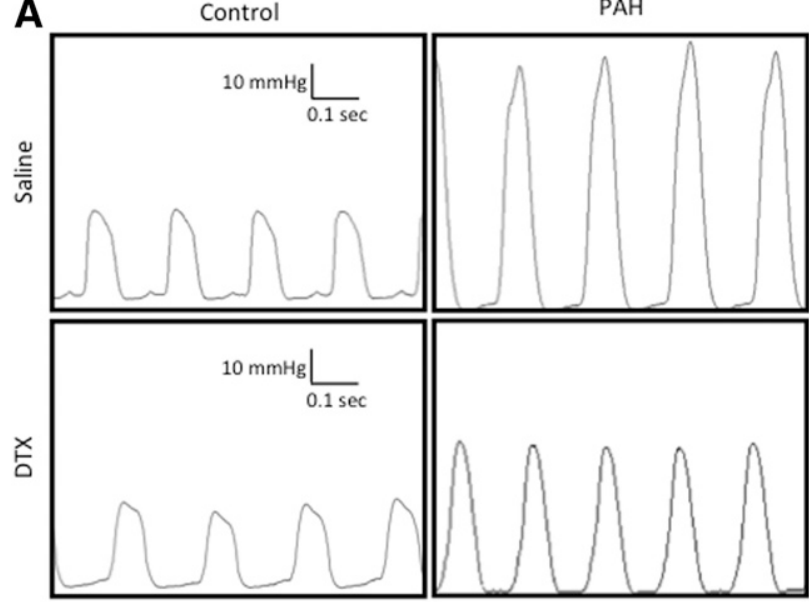

C

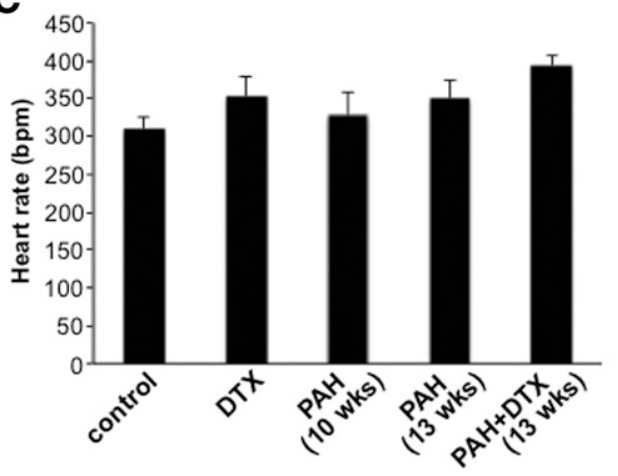

B

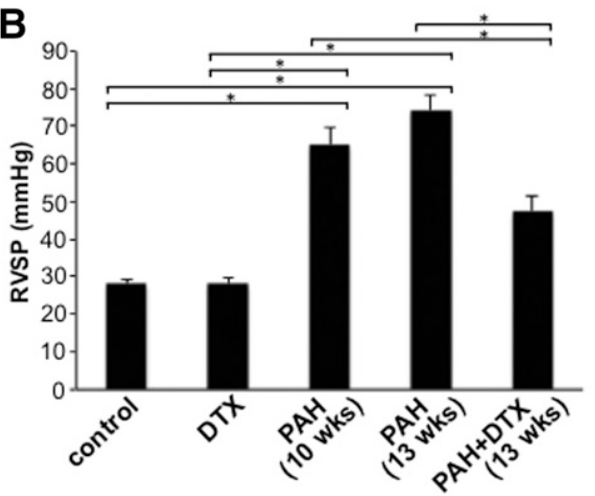

D

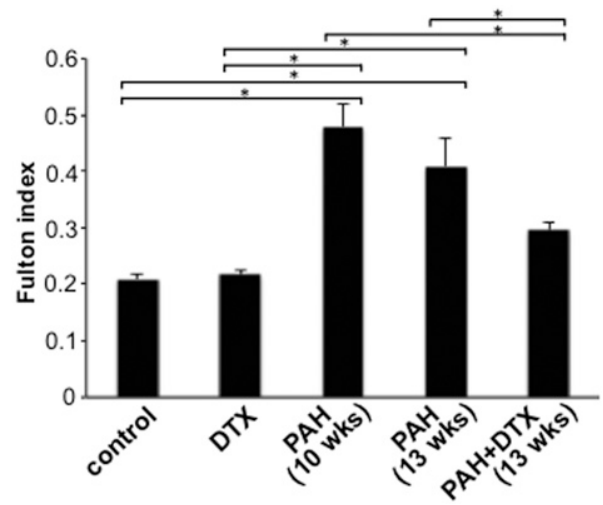

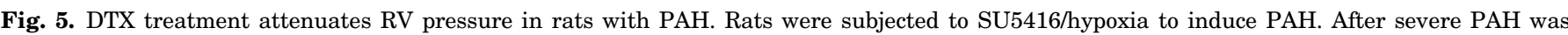

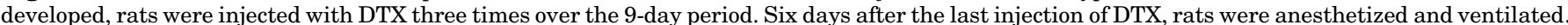

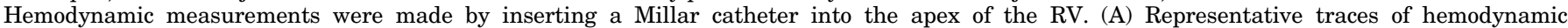

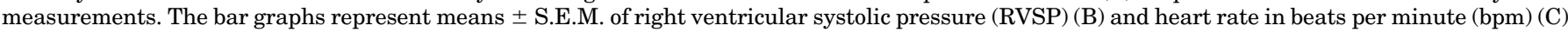

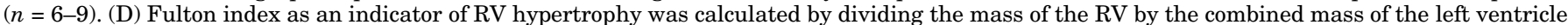
plus the septum. *Significant difference from each other at $P<0.05(n=6)$.

PASMCs was found to be similar to another member of the taxane family, paclitaxel (Fig. 1E), as well as to vincristine (Fig. 1F), another antitumor drug that disrupts microtubules with a different mechanism of inhibiting microtubular polymerization. DTX also effectively caused the death of proliferating human PAECs as determined by a cell viability assay (Fig. 2A) as well as by cell counting (Fig. 2B). Figure 2C shows representative photographs of control PAECs and cells treated with DTX.

DTX Effectively Reverses Pulmonary Vascular Remodeling. Based on these results in cultured pulmonary vascular cells, we tested the effects of DTX in an in vivo model of PAH. The injection of SU5416 into rats followed by subjecting the animals to sustained hypoxia for 3 weeks and subsequently maintaining them in normoxia resulted in the development of severe PAH and pulmonary vascular remodeling (Oka et al., 2007).

H\&E stain and immunohistochemistry with the $\alpha$-smooth muscle actin antibody showed that, in contrast to the normal control lung, the majority of PA walls are thickened and most of the small caliber vessels are occluded in the lungs of rats with PAH. DTX treatment of PAH animals decreased the PA medial wall thickness, reduced the expression of $\alpha$-smooth muscle actin, and increased the lumen area (Fig. 3A). The quantifications of the wall thickness of pulmonary arterioles/ small PAs indicated DTX completely reversed the PA wall thickening of SU5416/hypoxia-treated rats to the normal level (Fig. 3B). The DTX treatment had no effects on normal PA in control rats, demonstrating the selectivity of DTX to remodeled pulmonary vasculatures.

The examinations of H\&E-stained slides also revealed that, while some severe pulmonary vascular remodeling lesions, such as concentric lamellae and plexiform lesions, were present in all rats with PAH (Fig. 4), we observed such lesions in only one out of six PAH rats treated with DTX.

This reversal of pulmonary vascular thickening was accompanied by a reduction in right ventricular systolic pressure (Fig. 5, A and B). Heart rate was not affected by the DTX treatment in either control rats or rats with PAH (Fig. 5C). The treatment of PAH rats with DTX reversed RV hypertrophy as revealed by calculating the Fulton Index (Fig. 5D).

Inhibition of Autophagy Mediates DTX-Induced Cell Death. Since our previous studies using other antitumor drugs (daunorubicin, bortezomib, and carfilzomib) showed the importance of autophagic cell death (Ibrahim et al., 2014; Wang et al., 2016), we tested if the DTX-induced death of PASMCs may be mediated by autophagy. Surprisingly, Fig. 6A shows that, similar to DTX, an autophagy inhibitor (SBI-0206965) reduced the number of proliferating/synthetic PASMCs, whereas an apoptosis inhibitor (Z-VAD-FMK) had no effects. Further, the results from the experiments in which 

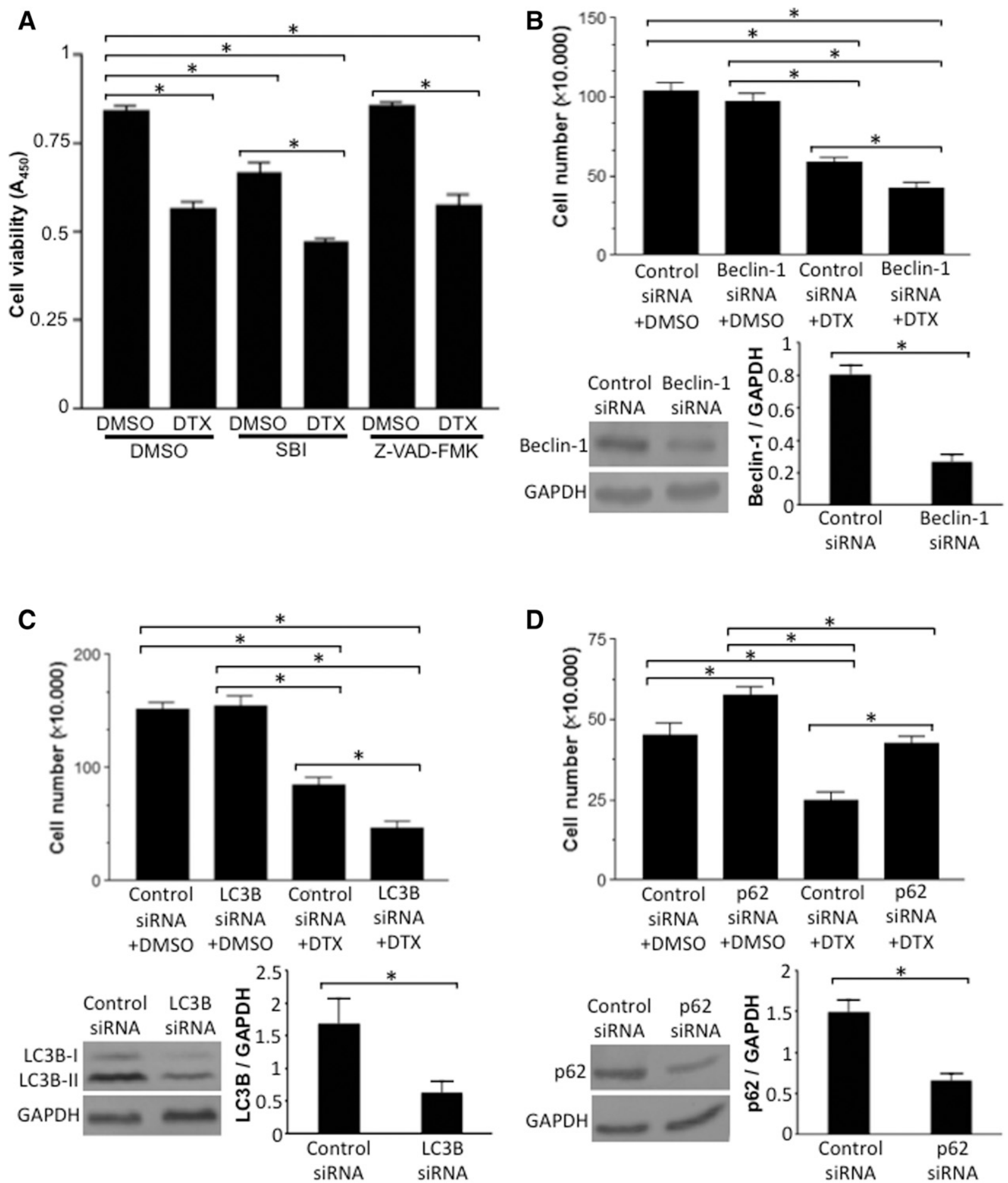

Fig. 6. Inhibition of autophagy potentiates DTX-induced death of PASMCs. (A) Proliferating/synthetic human PASMCs were pretreated with dimethylsulfoxide (DMSO; 0.5\%), SBI-0206965 (50 $\mu \mathrm{M})$, or Z-VAD-FMK (50 $\mu \mathrm{M})$ for 30 minutes and then treated with DMSO $(0.1 \%)$ or DTX (50 $\mathrm{nM})$ for 22 hours. The number of viable cells was monitored by using Cell Counting Kit-8 at absorbance $450 \mathrm{~nm}\left(\mathrm{~A}_{450}\right)$. (B) Cells were transfected with siRNA for beclin-1 or control scrambled siRNA for 2 days. Cells were then treated with DMSO or DTX ( $50 \mathrm{nM}$ ) for 22 hours, and cell number was counted using a hemocytometer. Western blotting results demonstrate the extent of siRNA knockdown of Beclin-1 $(n=6-9)$. (C) Cells were transfected with siRNA for LC3B or control scrambled siRNA. Cells were then treated with DMSO or DTX, and cell number was counted. Western blotting results demonstrate the extent of siRNA knockdown of LC3B $(n=5)$. (D) Cells were transfected with siRNA for p62 or control scrambled siRNA. Cells were then treated with DMSO or DTX, and cell number was counted. Western blotting results demonstrate the extent of siRNA knockdown of p62 $(n=6-9)$. GAPDH, glyceraldehyde-3-phosphate dehydrogenase.

cells were transfected with siRNA for various autophagy regulatory proteins showed that knocking down mediators of autophagy, Beclin-1 (Fig. 6B) and LC3B (Fig. 6C), enhanced DTX-induced cell death. By contrast, knocking down p62, which is downregulated during the autophagic process, reduced DTX-induced cell death (Fig. 6D). These results suggest that the DTX-induced death of PASMCs is dependent on the inhibition of the cell survival role of autophagy. 

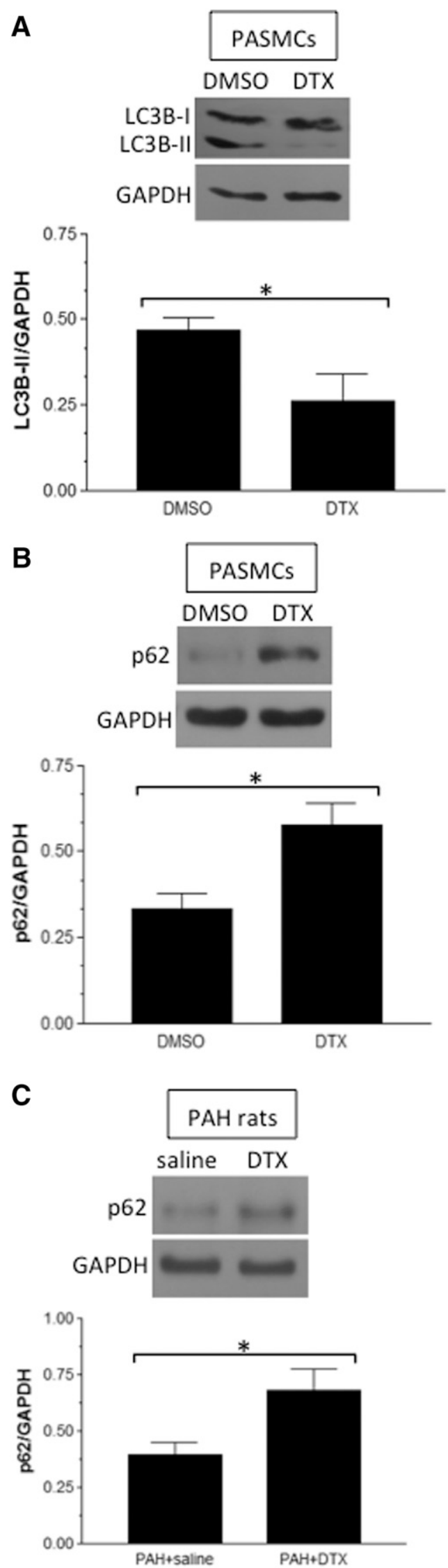

Fig. 7. Effects of DTX on autophagy in PASMCs. (A and B) Human PASMCs were treated with dimethylsulfoxide (DMSO; $0.1 \%$ ) or DTX (50 nM) for 22 hours, and cell lysates were subjected to Western blotting to
In contrast to previous studies, in which anthracycline and proteasome inhibitors promoted autophagy as monitored by the formation of LC3B-II and the downregulation of p62 in remodeled pulmonary vascular smooth muscle (Ibrahim et al., 2014; Wang et al., 2016), we found that DTX reduced the LC3B-II level (Fig. 7A) and increased p62 expression (Fig. 7B) in human PASMCs. In PAs from rats with PAH, DTX administration increased the p62 level (Fig. 7C). These results revealed that, in remodeled PASMCs with the proliferating phenotype, DTX decreases the activity of autophagy.

DTX Inhibits Autophagy by Promoting the Degradation of Beclin-1. A mechanism of the DTX-induced inhibition of autophagy appears to involve the downregulation of Beclin-1 protein expression, as DTX decreases Beclin-1 protein expression in both cultured human PASMCs (Fig. 8A) and the PAs of rats with PAH (Fig. 8B). The beclin-1 mRNA expression as monitored by reverse-transcription polymerase chain reaction was not affected by the DTX treatment of cultured PASMCs (Fig. 8C). Further, Beclin-1 ectopically expressed via adenovirusmediated gene transfer driven by the CMV (cytomegalovirus) promoter was also downregulated by DTX (Fig. 8D).

Thus, we hypothesized that DTX drives the degradation of Beclin-1 protein. Experiments using a proteasome inhibitor support this hypothesis, as the pretreatment of PASMCs with MG132 inhibited the DTX-induced decrease of Beclin-1 protein expression (Fig. 8E). These results demonstrated that DTX promotes the proteasome-dependent degradation of Beclin-1 that, in turn, reduces the formation of LC3B-II and enhances p62 accumulation, leading to cell death.

DTX Promotes Interactions of Beclin-1 with MYH9, Which Participates in Cell Death. To explore the mechanism of the DTX regulation of Beclin-1, we searched for proteins that bind to Beclin-1 in response to the DTX treatment. Human PASMCs were treated with or without DTX, cell lysates were prepared, and samples were immunoprecipitated with the rabbit Beclin-1 antibody and subjected to SDS-PAGE followed by Coomassie Blue staining. We found a band between 150 and $250 \mathrm{kDa}$ to be consistently higher in DTX-treated cells (Fig. 9A). This band was not generated when immunoprecipitation was performed with normal rabbit IgG. Mass spectrometry identified that this band contains MYH9 (nonmuscle myosin heavy chain 9; accession P35579). The immunoprecipitation of human PASMC lysates with the Beclin-1 antibody followed by Western blotting with the MYH9 antibody also demonstrated that DTX increased the interactions between these two proteins (Fig. 9B). Similarly, the immunoprecipitation of PA homogenates from rats treated with DTX using the MYH9 antibody followed by Western blotting with the Beclin-1 antibody also showed this DTX-mediated event (Fig. 9C).

To assess the function of MYH9 in the regulation of DTX actions on cell death, MYH9 was knocked down in proliferating/ synthetic human PASMCs by using siRNA (Fig. 9D). Knocking down MYH9 suppressed the DTX-induced cell death (Fig. 9E).

monitor LC3B-II and p62 levels $(n=5-7)$. (C) Rats were treated with SU5416/hypoxia and injected with saline or DTX. Protein levels of p62 were monitored by Western blotting in isolated PA homogenates $(n=7)$. *Significant difference between each other at $P<0.05$. GAPDH, glyceraldehyde-3-phosphate dehydrogenase. 
A
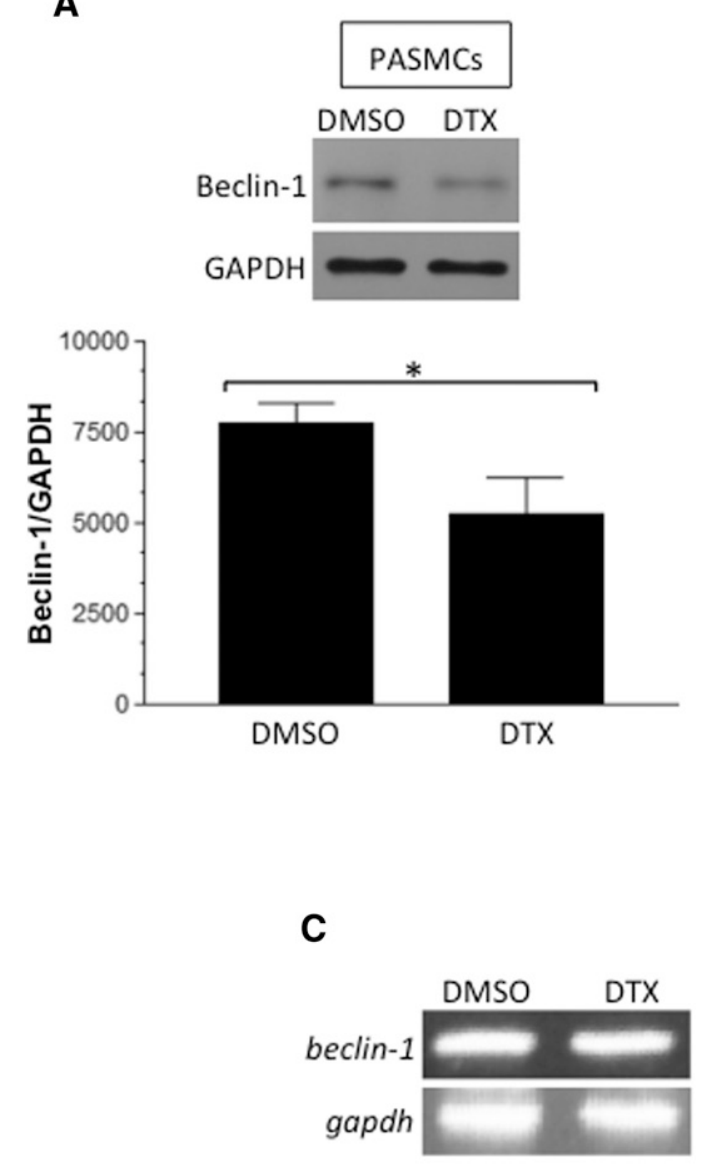

D
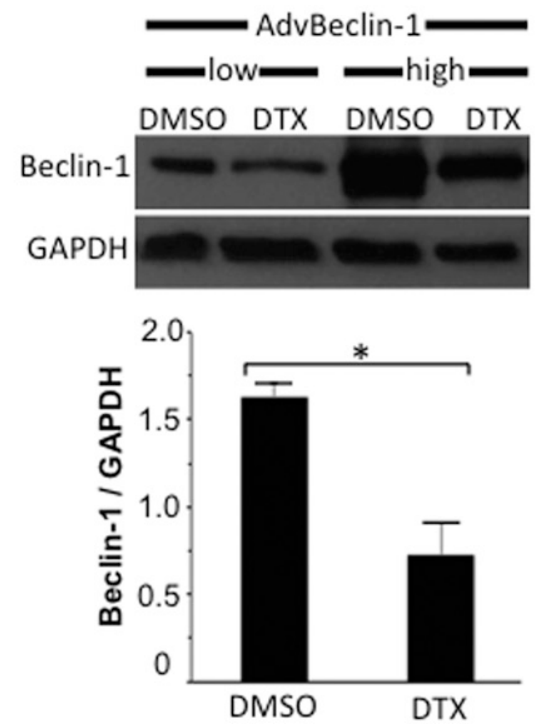

B

Reversal of PAH and RV Failure by Docetaxel
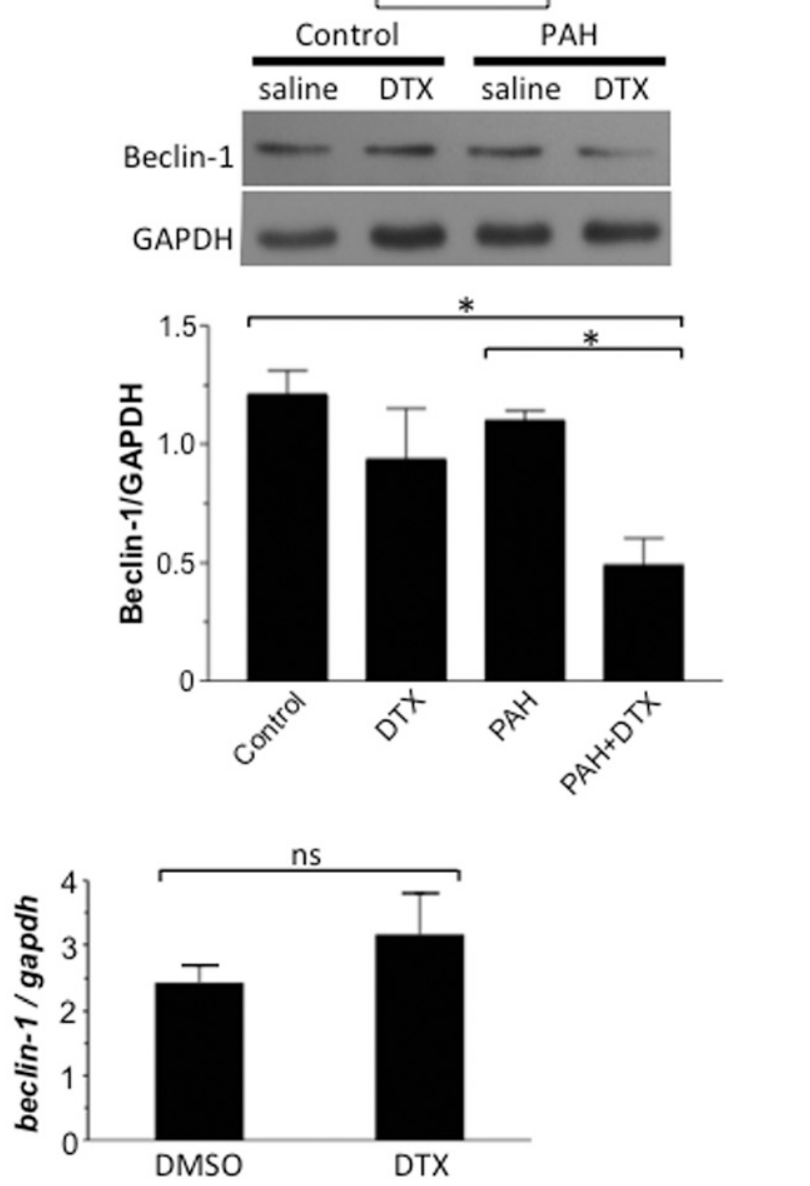

E

MG132 MG132
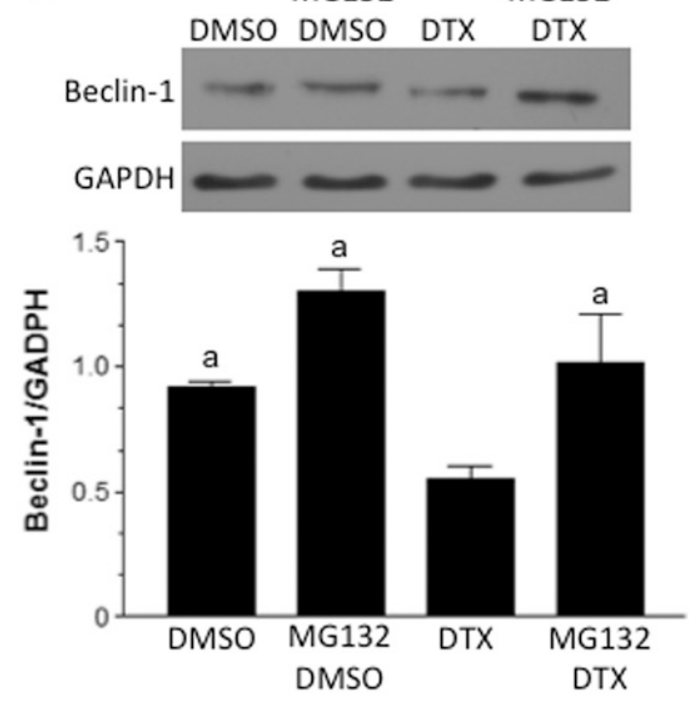

Fig. 8. Effects of DTX on Beclin-1. (A) Proliferating/synthetic human PASMCs were treated with dimethylsulfoxide (DMSO; 0.1\%) or DTX (50 nM) for 22 hours. Beclin-1 protein expression was monitored by Western blotting $(n=6)$. *Values that are significantly different from each other at $P<0.05$. (B) Rats with PAH and control rats were treated with saline or DTX, and Beclin-1 protein expression was monitored in the homogenates of isolated PAs $(n=7)$. *Values that are significantly different from each other at $P<0.05$. (C) Proliferating/synthetic human PASMCs were treated with DMSO or DTX (50 nM) for 22 hours. The beclin-1 mRNA expression was monitored by reverse-transcription polymerase chain reaction $(n=6)$. ns, values are not significantly different from each other at $P<0.05$. (D) Human PASMCs were infected with adenovirus expressing Beclin- 1 for 48 hours. Cells were then treated with DMSO (0.1\%) or DTX $(50 \mathrm{nM})$ for 22 hours. Beclin-1 protein expression was monitored by Western blotting $(n=3)$. *Values that are significantly different from each other at $P<$ 0.05. (E) Human PASMCs were pretreated with MG132 (250 nM) for 6 hours and then treated with DMSO (0.1\%) or DTX (50 nM) for 22 hours. Beclin-1 protein expression was monitored by Western blotting in cell lysates $(n=6)$. The symbol "a" denotes values that are significantly different from the DTX value at $P<0.05$. GAPDH, glyceraldehyde-3-phosphate dehydrogenase. 


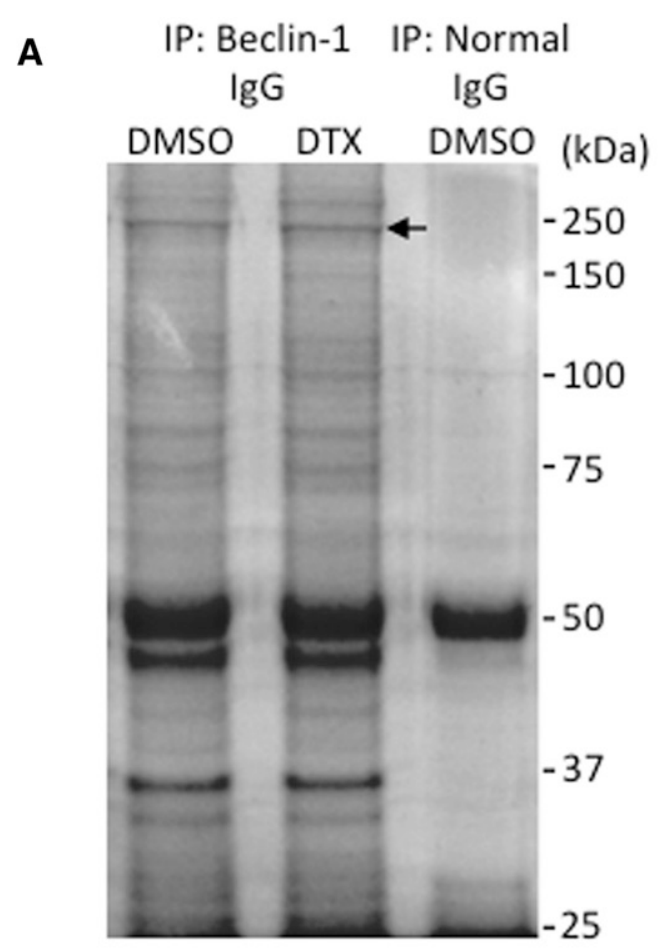

C
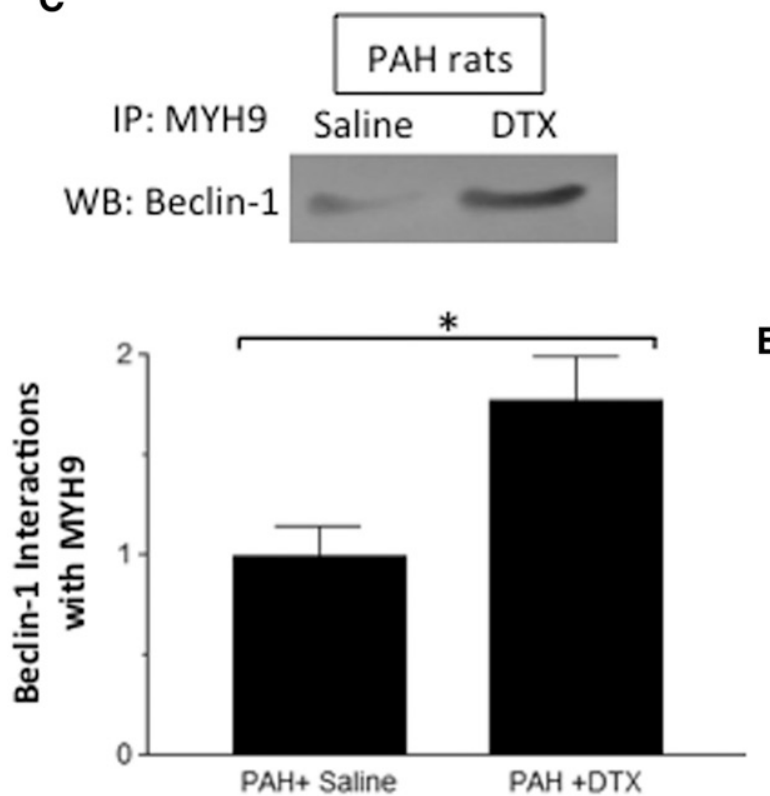
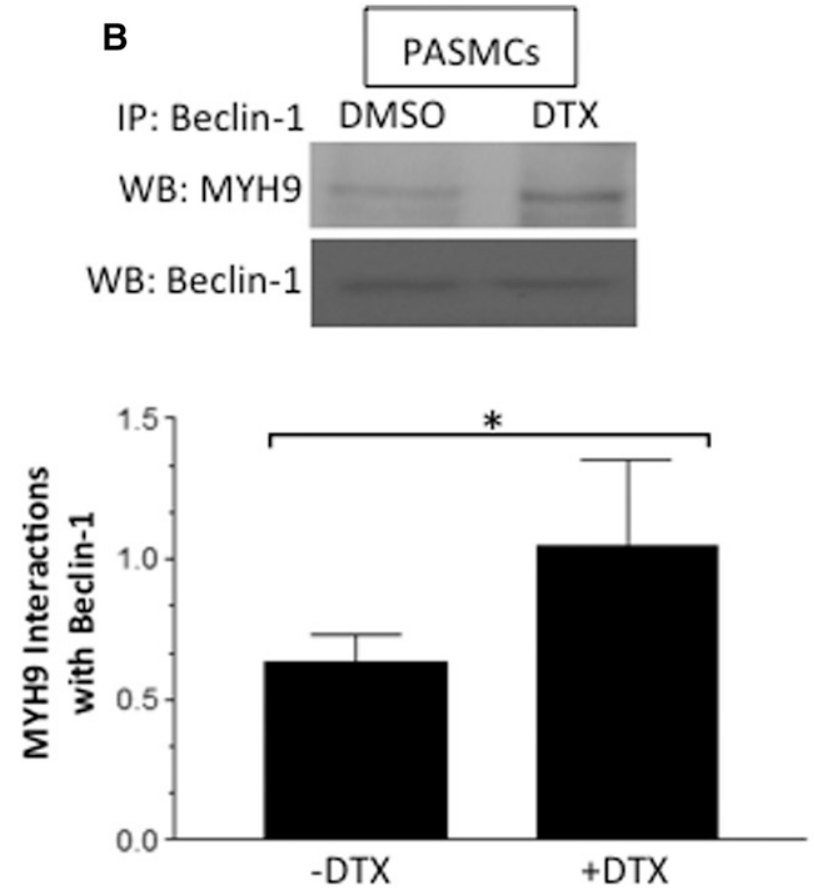

D
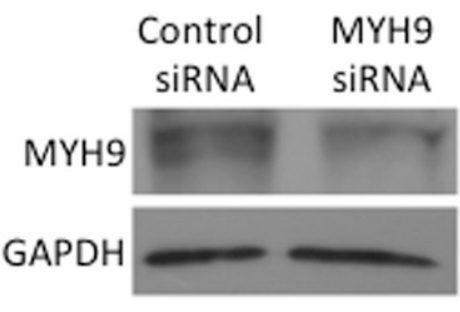

E

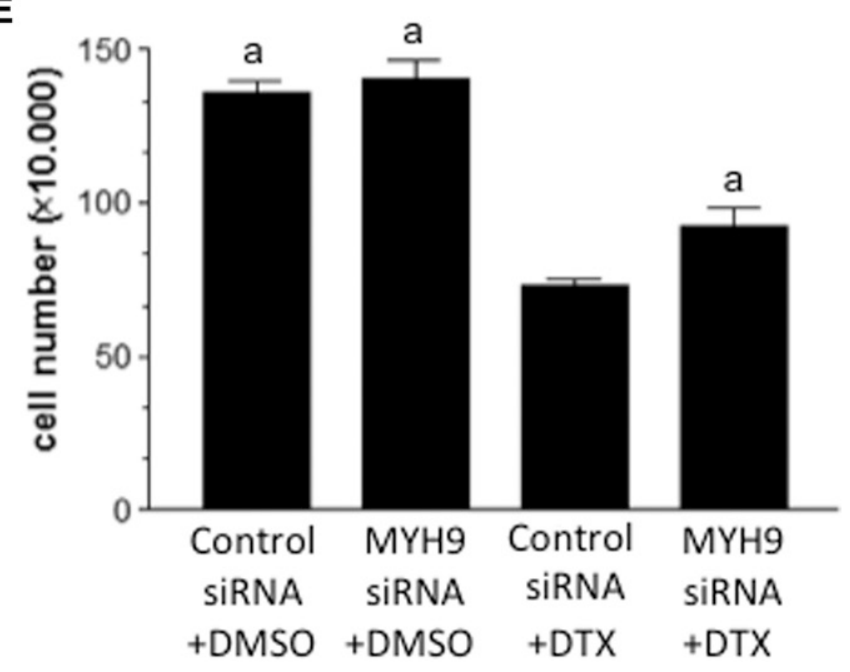

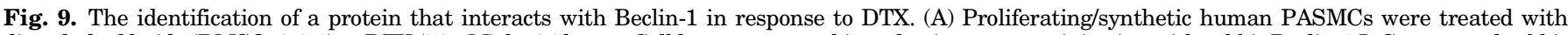

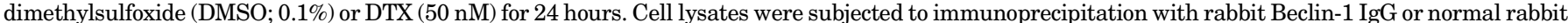

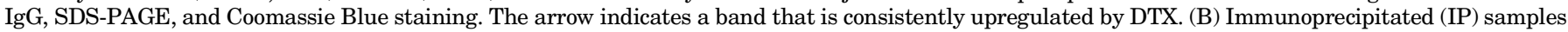

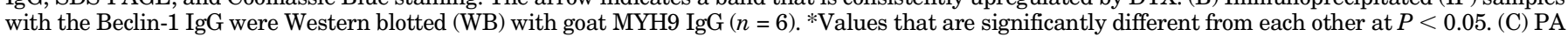

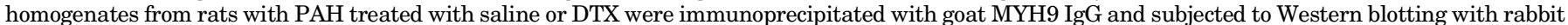

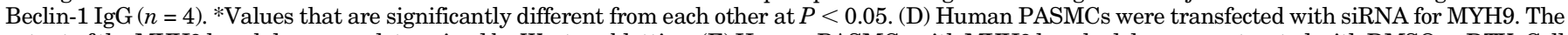

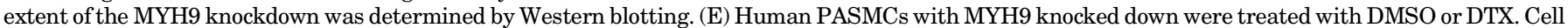

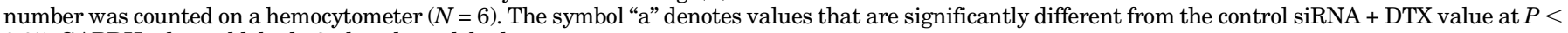
0.05 . GAPDH, glyceraldehyde-3-phosphate dehydrogenase. 
A
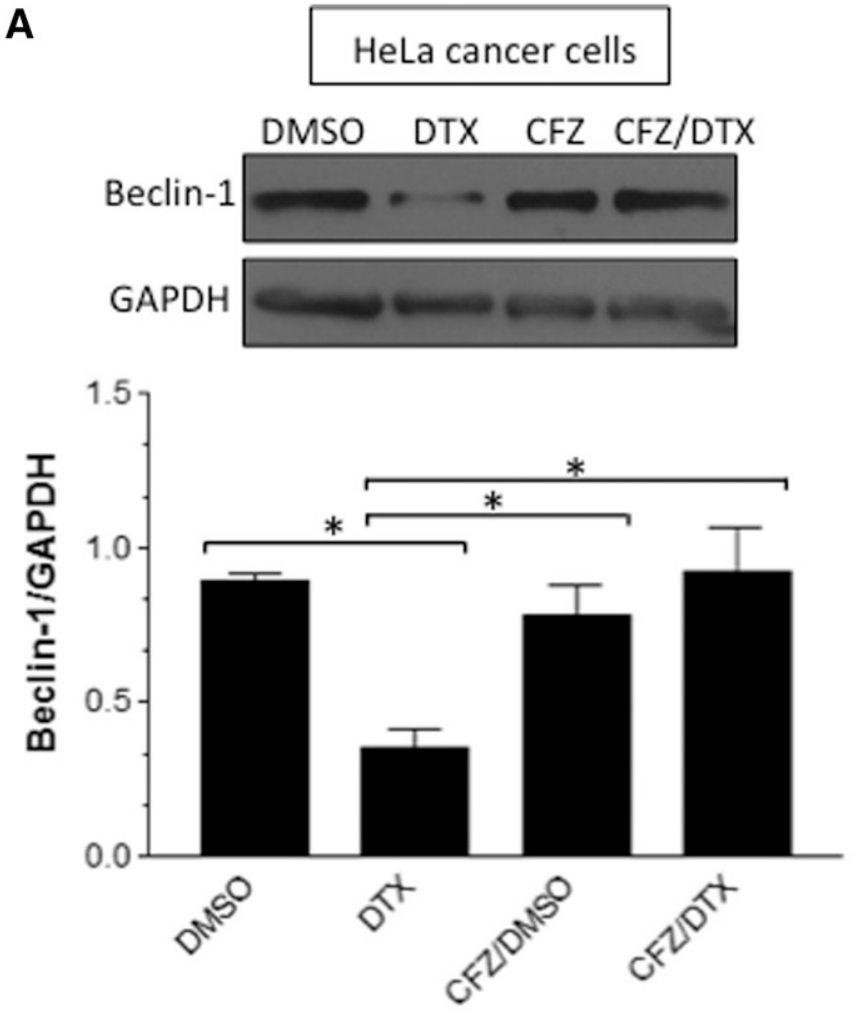

B
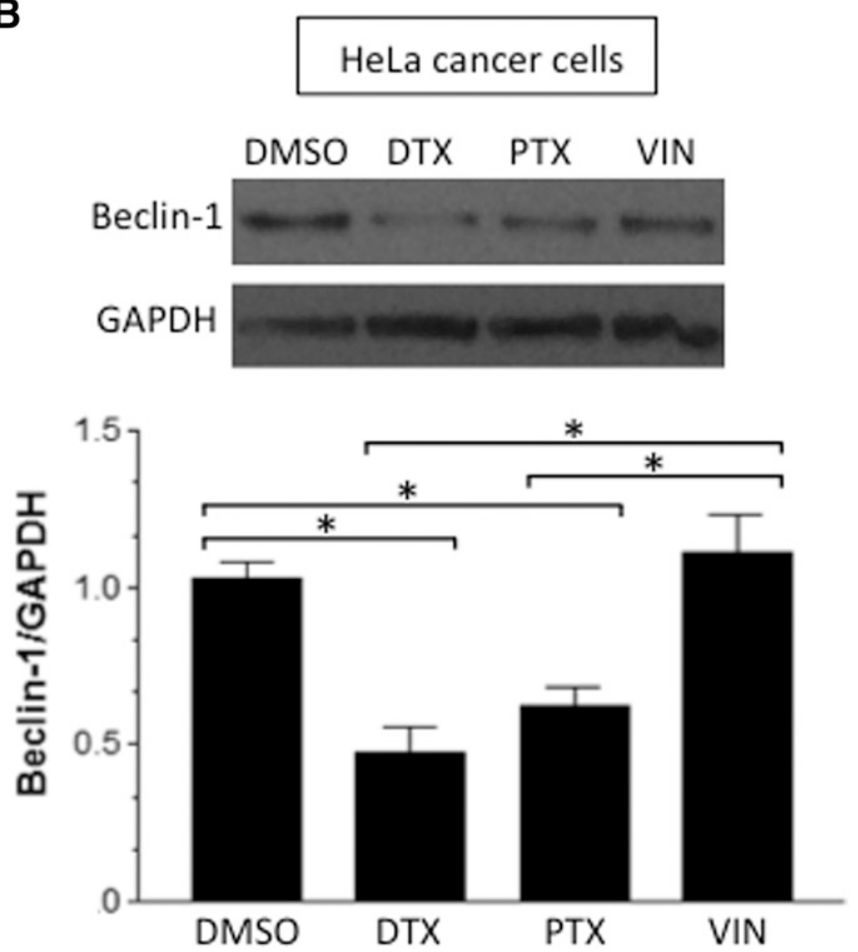

Fig. 10. DTX-induced downregulation of Beclin-1 in cancer cells. (A) HeLa cells were pretreated with dimethylsulfoxide (DMSO; $0.1 \%$ ) or carfilzomib (CFZ; $300 \mathrm{nM}$ ) for 30 minutes, then treated with DMSO $(0.1 \%)$ or DTX (50 nM) for 24 hours. (B) HeLa cells were treated with DMSO, DTX, paclitaxel (PTX), or vincristine (VIN) for 24 hours. Beclin-1 protein expression was monitored by Western blotting in cell lysates $(n=4)$. *Values that are significantly different from each other at $P<0.05$. GAPDH, glyceraldehyde-3-phosphate dehydrogenase.
These results support the concept that DTX promotes the death of PASMCs by inhibiting autophagy through the proteasomal degradation of Beclin-1 that is regulated by MYH9.

DTX Also Downregulates Beclin-1 in Cancer Cells. To our knowledge, the mechanism of DTX action that mediates the Beclin-1 degradation has not been reported, even in cancer cells. Thus, we tested if the mechanism found in PASMCs may also be operative in cancer cells. As shown in Fig. 10A, treating HeLa human cervical cancer cells with DTX resulted in the downregulation of Beclin-1. Further, this effect was inhibited by a proteasome inhibitor, carfilzomib. Carfilzomib alone did not affect Beclin-1 protein expression. The downregulation of Beclin-1 also occurred with another taxane drug, paclitaxel, but not by a nontaxane modulator of microtubules, vincristine (Fig. 10B), indicating the specificity of this mechanism to taxanes.

Effects of DTX on the Heart. Antitumor agents have the potential to exert toxicity, in particular, cardiotoxicity (Albini et al., 2010; Minotti et al., 2010). Since PAH patients already have failing RVs, the use of antitumor drugs would be a great clinical concern. The H\&E-staining results shown in Fig. 11A demonstrate that the RVs of control rats have cardiomyocytes in parallel with elongated and centrally located nuclei and intercalated discs. On the other hand, the RVs of rats with PAH exhibit microfocal alterations of cardiomyocytes (black arrows shown in $\times 200$ ), congestion of the microvasculature, dilated vessels, mild interstitial edema, hypertrophy and atrophy of cardiomyocytes, cell hypereosinophilia (blue arrow shown in $\times 200$ ), contracture, transverse bands of dense eosinophilic materials divided by a pale structure with a frequent finely granular or vacuolated sarcoplasm, indistinct cross-striations of cardiomyocytes, and myofibrils having variable extent of dissolutions (myofibrillar lysis) (black arrows shown in $\times 200)$. A higher magnification $(\times 400)$ also revealed that the RVs of $\mathrm{PAH}$ rats have mild perinuclear and intermyofibrillar edema (green arrow in $\times 400$ ), wavy arrangement of cardiomyocyte fibers (black arrow shown in $\times 400$ ), cytoplasmic granules of the cardiomyocytes (blue arrows shown in $\times 400$ ), and focal myocytolysis. All of these lesions seen in the RVs of PAH rats were either absent or markedly less pronounced in the RVs of PAH rats treated with DTX. Specifically, cardiomyocytes are arranged in parallel with clear cross-striations as in control healthy rats, and characteristics observed in PAH RVs, including intermyofibrillar and perinuclear edema, the wavy arrangement of cardiomyocytes, the contracture of cardiomyocytes, myofibrillar lesions, and atrophied myocytes, were all absent. However, some hypertrophied cardiomyocytes were still observed in these DTX-treated PAH RVs.

TUNEL staining demonstrated that apoptotic cardiomyocytes are present in the RVs of PAH rats (Fig. 11, A and B). On the other hand, the number of TUNEL-positive cells is significantly less in the RVs of PAH rats treated with DTX. Western blotting monitoring cleaved caspase-3 confirmed that the apoptosis is promoted in the RV by PAH, and DTX reduces the degree of apoptosis (Fig. 12A). Similar results were obtained in the left ventricle (data not shown).

Remarkably, Masson's trichrome stain revealed that DTX resolved the RV fibrosis that developed in response to PAH (Fig. 11A). The quantifications of Masson's trichrome stain indeed determined that DTX significantly reduced the extent of RV fibrosis (Fig. 11C). 


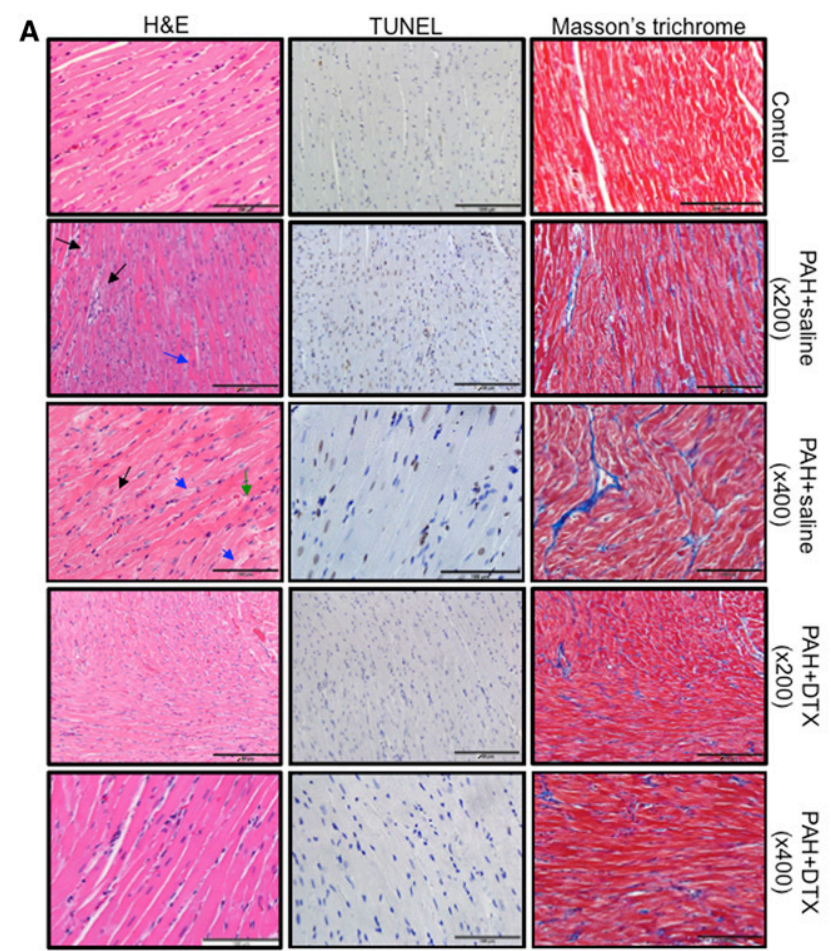

Functionally, hemodynamic measurements indicated that the contractility index of the RV improved following the DTX treatment of $\mathrm{PAH}$ rats (Fig. 12B).

These results demonstrated that DTX does not exert cardiotoxicity; rather, it exerts protective effects in the RV affected by PAH.

\section{Discussion}

$\mathrm{PAH}$ is an aggressive disease with a high mortality rate (Thenappan et al., 2007). The progressive nature of this disease as well as the absence of a satisfactory curative treatment raise a pressing need to investigate its underlying molecular mechanisms and provide novel therapeutic regiments for those suffering from PAH. The uncontrolled intimal and medial growth of the small pulmonary arterioles in this disease is a main trigger for the dramatic increase in PA pressure that results in $\mathrm{RV}$ failure. Therefore, allowing pharmacologic cell death for these unnecessary proliferating cells should ameliorate the underlying pulmonary vascular remodeling and alleviate the stress to the $R V$.

In our previous studies (Ibrahim et al., 2014; Wang et al., 2016), antitumor drugs including an anthracycline (daunorubicin) and proteasome inhibitors (MG132, bortezomib, carfilzomib) were found to effectively promote the death of pulmonary vascular cells and reverse pulmonary vascular remodeling. The actions of these drugs to reduce PA wall thickness were specific to remodeled vessels without affecting the normal pulmonary vasculature. The administration of these antitumor drugs alone, however, did not influence RV pressure. Rather, we found that these antitumor agents are capable of potentiating the ability of vasodilators to reduce $\mathrm{RV}$ pressure. Thus, we proposed that these cell-killing agents could be used in combination with already used vasodilators to establish better therapeutic strategies for treating PAH.

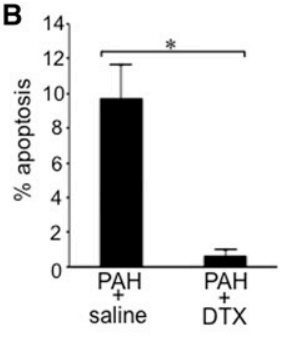

Fig. 11. Effects of DTX on the RV of rats with PAH. Rats were subjected to SU5416/hypoxia to promote PAH. After severe PAH was developed, rats were injected with saline or DTX three times

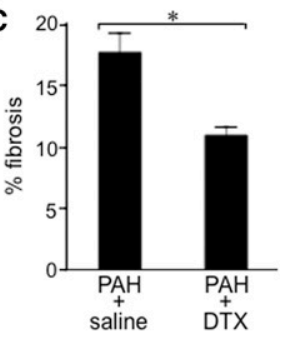
over the 9-day period. Six days after the last injection of DTX, hearts were harvested for histology analysis. (A) Representative results of H\&E stain, TUNEL assay, and Masson's trichrome stain in the RVs are shown. (B) Percentage apoptosis in the RVs was determined in TUNELstained slides. (C) Percentage fibrosis in the RVs was determined in Masson's trichrome-stained slides. *Values significantly different from each other at $P<0.05(n=4)$.
However, like many cancers, since PAH patients have short survival durations, one must decide if the benefit of aggressive therapies using antitumor drugs outweighs various toxicities exerted by these drugs. One serious concern with treating $\mathrm{PAH}$ patients with antitumor drugs to kill excess pulmonary vascular cells is that many of these agents may also kill cardiac muscle cells (Albini et al., 2010; Minotti et al., 2010), promoting cardiotoxicity. Since the RVs of PAH patients have already been weakened by pressure overload, the potential impact of antitumor agents on the cardiac musculature needs to be carefully assessed. In fact, recent studies have demonstrated that proteasome inhibitors caused cardiac apoptosis in rats with PAH (Kim et al., 2012; Wang et al., 2016).

We questioned if other antitumor agents might have similar efficacies, but fewer cardiac concerns. We screened different cancer chemotherapeutic agents to mediate the cell killing of proliferating human PASMCs. Our data showed that DTX, a microtubule inhibitor, had an equal cell-killing efficacy to anthracycline (daunorubicin) and proteasome inhibitors (MG132, bortezomib). Other agents, including methotrexate (an antifolate) and ifosfamide (a nitrogen mustard alkylating agent), did not show significant cell-killing abilities. Gemcitabine (a nucleoside analog) was also effective at killing proliferating PASMCs, but this was the only drug capable of killing the differentiated/contractile phenotype as well. Thus, gemcitabine likely adversely affects the functional PASMCs needed for muscle contraction and vasoregulation.

We therefore tested the effects of DTX on an in vivo model of $\mathrm{PAH}$ with pathogenic features similar to those in human patients. The standard schedule of DTX administration in human cancer is $100 \mathrm{mg} / \mathrm{m}^{2}$; this is equivalent to $20 \mathrm{mg} / \mathrm{kg}$ body weight in rats. In the present study, we administered DTX to rats at a total dose of $16 \mathrm{mg} / \mathrm{kg}$ body weight, which is a dose less than half of the doses previously reported in in vivo cancer studies (Chan et al., 2002; Otová et al., 2006). Further, 
A
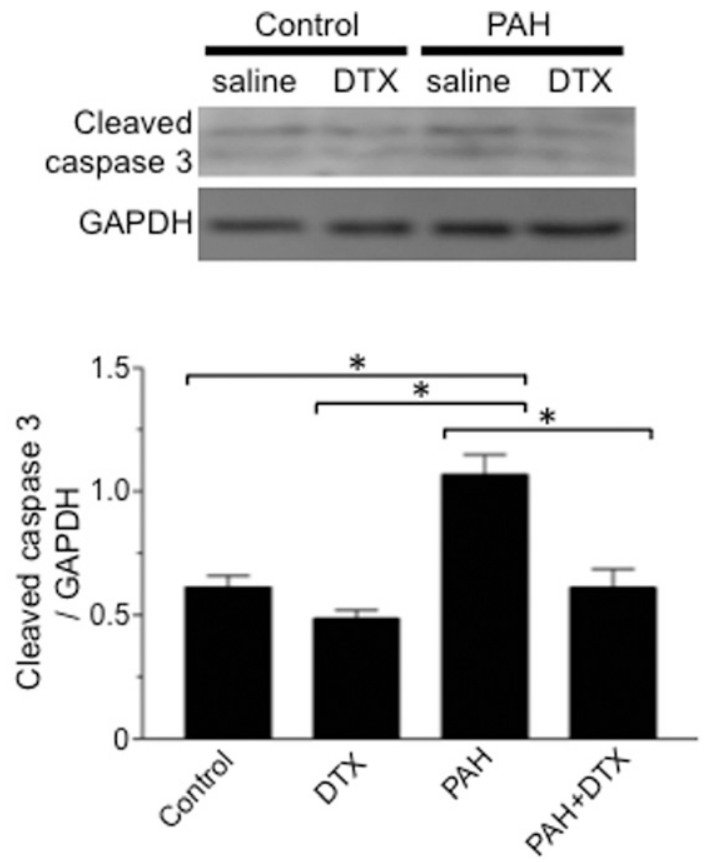

B

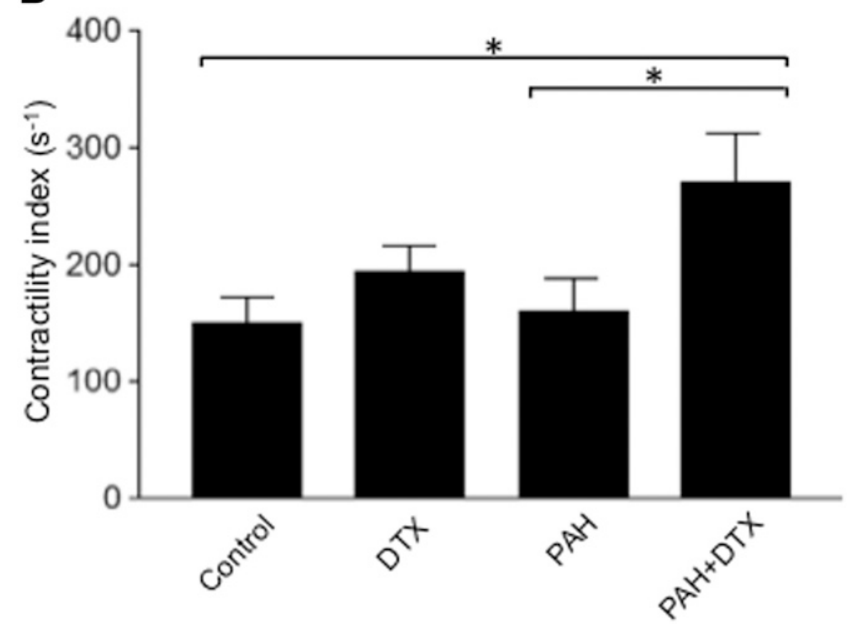

Fig. 12. Effects of DTX on cleaved caspase-3 expression and contractility index in rat RVs. Rats were subjected to SU5416/hypoxia treatment to promote PAH. After severe PAH was developed, rats were injected with saline or DTX three times over the 9-day period. (A) Six days after the last injection of DTX, hearts were harvested. The expression of cleaved caspase-3 was monitored by Western blotting in RV homogenates $(n=$ 4-5). (B) Six days after the last injection of DTX, rats were anesthetized and ventilated. Hemodynamic measurements were made by inserting a Millar catheter into the apex of the RV. The bar graph represents contractility index that was calculated by dividing $\mathrm{dP} / \mathrm{dt}_{\max }$ by the pressure at the time of $\mathrm{dP} / \mathrm{dt}_{\max }(n=6)$. *The values are significantly different from each other at $P<0.05$. GAPDH, glyceraldehyde-3phosphate dehydrogenase.

our cell culture experiments were conducted by using a DTX dose in the therapeutic range (Hernández-Vargas et al., 2007).

The present study revealed that therapeutic doses of DTX reversed pulmonary vascular remodeling and killed PASMCs and PAECs. The effects of DTX to reduce vascular wall thickness were limited to the PAs of animals with $\mathrm{PAH}$, whereas no reduction of normal vessels was noted, indicating the selectivity of this drug. In addition, DTX exhibited superior outcomes compared with other antitumor agents we have previously tested (Ibrahim et al., 2014; Wang et al., 2016) in that DTX reduced the RV damage caused by PAH. It is remarkable that the already-existing myocyte deterioration and already-developed severe fibrosis in the RV of PAH rats were replaced with the functional myocardium by the DTX treatment.

The mechanism of DTX-induced PASMC killing was also found to be different from those of anthracycline and proteasome inhibitors, which mediate autophagic cell death (Ibrahim et al., 2014; Wang et al., 2016). We initially hypothesized that autophagy mediates the cell-killing effects of DTX. Thus, we evaluated the protein expression of Beclin-1, LC3B-II, and p62. Surprisingly, in contrast to anthracycline and proteasome inhibitors, DTX caused the downregulation of LC3B-II and the upregulation of $\mathrm{p} 62$, indicating that the autophagy mechanism is inhibited. We have further provided evidence that this inhibition of the autophagy mechanism is through the proteasome-dependent degradation of Beclin-1, which may be regulated by MYH9. Since autophagy can also serve as a programmed cell survival mediator (Baehrecke, 2005), autophagy may be essential to maintain the survival of PASMCs, and DTX appears to target this mechanism for mediating cell death.

In summary, the present study reports that a taxane cancer chemotherapeutic agent, DTX, effectively attenuates pulmonary vascular remodeling in pulmonary hypertension. Not only does DTX not cause apparent cardiotoxicity, this drug actually repairs the RV damage induced by PAH. DTX also utilizes a unique mechanism of cell death compared with other antitumor agents (Ibrahim et al., 2014; Wang et al., 2016) by suppressing the cell survival role of autophagy. We propose that DTX may be useful to treat human $\mathrm{PAH}$ and right heart failure.

\section{Authorship Contributions}

Participated in research design: Ibrahim, Shults, Suzuki.

Conducted experiments: Ibrahim, Shults, Rybka, Suzuki.

Performed data analysis: Ibrahim, Shults, Suzuki.

Wrote or contributed to the writing of the manuscript: Ibrahim, Shults, Suzuki.

\section{References}

Abe K, Toba M, Alzoubi A, Ito M, Fagan KA, Cool CD, Voelkel NF, McMurtry IF, and Oka M (2010) Formation of plexiform lesions in experimental severe pulmonary arterial hypertension. Circulation 121:2747-2754.

Albini A, Pennesi G, Donatelli F, Cammarota R, De Flora S, and Noonan DM (2010) Cardiotoxicity of anticancer drugs: the need for cardio-oncology and cardiooncological prevention. J Natl Cancer Inst 102:14-25.

Archer SL and Michelakis ED (2006) An evidence-based approach to the management of pulmonary arterial hypertension. Curr Opin Cardiol 21:385-392.

Baehrecke EH (2005) Autophagy: dual roles in life and death? Nat Rev Mol Cell Biol 6:505-510.

Benza RL, Miller DP, Frost A, Barst RJ, Krichman AM, and McGoon MD (2010) Analysis of the lung allocation score estimation of risk of death in patients with pulmonary arterial hypertension using data from the REVEAL Registry. Transplantation 90:298-305.

Bockorny M, Chakravarty S, Schulman P, Bockorny B, and Bona R (2012) Severe heart failure after bortezomib treatment in a patient with multiple myeloma: a case report and review of the literature. Acta Haematol 128:244-247.

Chan DC, Earle KA, Zhao TL, Helfrich B, Zeng C, Baron A, Whitehead CM, Piazza G, Pamukcu R, Thompson WJ, et al. (2002) Exisulind in combination with docetaxel inhibits growth and metastasis of human lung cancer and prolongs survival in athymic nude rats with orthotopic lung tumors. Clin Cancer Res 8:904-912.

Chung L, Domsic RT, Lingala B, Alkassab F, Bolster M, Csuka ME, Derk C, Fischer A, Frech T, Furst DE, et al. (2014) Survival and predictors of mortality in systemic sclerosis-associated pulmonary arterial hypertension: outcomes from the pulmonary hypertension assessment and recognition of outcomes in scleroderma registry. Arthritis Care Res (Hoboken) 66:489-495

D'Alonzo GE, Barst RJ, Ayres SM, Bergofsky EH, Brundage BH, Detre KM, Fishman AP, Goldring RM, Groves BM, Kernis JT, et al. (1991) Survival in patients with primary pulmonary hypertension. Results from a national prospective registry. Ann Intern Med 115:343-349.

Fojo T and Menefee M (2007) Mechanisms of multidrug resistance: the potential role of microtubule-stabilizing agents. Ann Oncol 18 (Suppl 5):v3-v8.

Galiè N, Hoeper MM, Humbert M, Torbicki A, Vachiery JL, Barbera JA, Beghetti M, Corris P, Gaine S, Gibbs JS, et al.; ESC Committee for Practice Guidelines (CPG) (2009) Guidelines for the diagnosis and treatment of pulmonary hypertension: the 
Task Force for the Diagnosis and Treatment of Pulmonary Hypertension of the European Society of Cardiology (ESC) and the European Respiratory Society (ERS), endorsed by the International Society of Heart and Lung Transplantation (ISHLT). Eur Heart $J$ 30:2493-2537.

Gligorov J and Lotz JP (2004) Preclinical pharmacology of the taxanes: implications of the differences. Oncologist 9 (Suppl 2):3-8.

Gupta A, Pandey A, and Sethi S (2012) Bortezomib-induced congestive cardiac failure in a patient with multiple myeloma. Cardiovasc Toxicol 12:184-187.

Hernández-Vargas H, Palacios J, and Moreno-Bueno G (2007) Molecular profiling of docetaxel cytotoxicity in breast cancer cells: uncoupling of aberrant mitosis and apoptosis. Oncogene 26:2902-2913.

Humbert M, Sitbon O, Yaïci A, Montani D, O’Callaghan DS, Jaïs X, Parent F, Savale L, Natali D, Günther S, et al.; French Pulmonary Arterial Hypertension Network (2010) Survival in incident and prevalent cohorts of patients with pulmonary arterial hypertension. Eur Respir J 36:549-555.

Ibrahim YF, Wong CM, Pavlickova L, Liu L, Trasar L, Bansal G, and Suzuki YJ (2014) Mechanism of the susceptibility of remodeled pulmonary vessels to druginduced cell killing. J Am Heart Assoc 3:e000520.

Jansa P, Jarkovsky J, Al-Hiti H, Popelova J, Ambroz D, Zatocil T, Votavova R, Polacek P, Maresova J, Aschermann M, et al. (2014) Epidemiology and long-term survival of pulmonary arterial hypertension in the Czech Republic: a retrospective analysis of a nationwide registry. BMC Pulm Med 14:45.

Kim SY, Lee JH, Huh JW, Kim HJ, Park MK, Ro JY, Oh YM, Lee SD, and Lee YS (2012) Bortezomib alleviates experimental pulmonary arterial hypertension. Am $J$ Respir Cell Mol Biol 47:698-708.

Menna P, Paz OG, Chello M, Covino E, Salvatorelli E, and Minotti G (2012) Anthracycline cardiotoxicity. Expert Opin Drug Saf 11 (Suppl 1):S21-S36.

Minotti G, Menna P, Salvatorelli E, Cairo G, and Gianni L (2004) Anthracyclines: molecular advances and pharmacologic developments in antitumor activity and cardiotoxicity. Pharmacol Rev 56:185-229.

Minotti G, Salvatorelli E, and Menna P (2010) Pharmacological foundations of cardiooncology. J Pharmacol Exp Ther 334:2-8.

Oka M, Homma N, Taraseviciene-Stewart L, Morris KG, Kraskauskas D, Burns N, Voelkel NF, and McMurtry IF (2007) Rho kinase-mediated vasoconstriction is important in severe occlusive pulmonary arterial hypertension in rats. Circ Res 100:923-929.
Olsson KM, Delcroix M, Ghofrani HA, Tiede H, Huscher D, Speich R, Grünig E, Staehler G, Rosenkranz S, Halank M, et al. (2014) Anticoagulation and survival in pulmonary arterial hypertension: results from the Comparative, Prospective Registry of Newly Initiated Therapies for Pulmonary Hypertension (COMPERA). Circulation 129:57-65.

Otová B, Václavíková R, Danielová V, Holubová J, Ehrlichová M, Horský S, Soucek P, Simek P, and Gut I (2006) Effects of paclitaxel, docetaxel and their combinations on subcutaneous lymphomas in inbred Sprague-Dawley/Cub rats. Eur J Pharm Sci 29:442-450.

Park AM, Wong CM, Jelinkova L, Liu L, Nagase H, and Suzuki YJ (2010) Pulmonary hypertension-induced GATA4 activation in the right ventricle. Hypertension 56: $1145-1151$.

Peacock AJ, Murphy NF, McMurray JJ, Caballero L, and Stewart S (2007) An epidemiological study of pulmonary arterial hypertension. Eur Respir $J$ 30:104-109.

Suzuki YJ, Nagase H, Wong CM, Kumar SV, Jain V, Park AM, and Day RM (2007) Regulation of Bcl- $\mathrm{x}_{\mathrm{L}}$ expression in lung vascular smooth muscle. Am J Respir Cell Mol Biol 36:678-687.

Taraseviciene-Stewart L, Kasahara Y, Alger L, Hirth P, Mc Mahon G, Waltenberger J, Voelkel NF, and Tuder RM (2001) Inhibition of the VEGF receptor 2 combined with chronic hypoxia causes cell death-dependent pulmonary endothelial cell proliferation and severe pulmonary hypertension. FASEB J 15:427-438.

Thenappan T, Shah SJ, Rich S, and Gomberg-Maitland M (2007) A USA-based registry for pulmonary arterial hypertension: 1982-2006. Eur Respir J 30:1103-1110.

Thenappan T, Shah SJ, Rich S, Tian L, Archer SL, and Gomberg-Maitland M (2010) Survival in pulmonary arterial hypertension: a reappraisal of the NIH risk stratification equation. Eur Respir J 35:1079-1087.

Thompson AA and Lawrie A (2017) Targeting vascular remodeling to treat pulmonary arterial hypertension. Trends Mol Med 23:31-45.

Wang X, Ibrahim YF, Das D, Zungu-Edmondson M, Shults NV, and Suzuki YJ (2016) Carfilzomib reverses pulmonary arterial hypertension. Cardiovasc Res 110:188-199.

Address correspondence to: Yuichiro J. Suzuki, Department of Pharmacology and Physiology, Georgetown University Medical Center, 3900 Reservoir Road NW, Washington, DC 20057. E-mail: ys82@georgetown.edu 